\title{
Mitochondrial mass, a new metabolic biomarker for stem-like cancer cells: Understanding WNT/FGF-driven anabolic signaling
}

\author{
Rebecca Lamb ${ }^{1,2, *}$, Gloria Bonuccelli, 2, ${ }^{1}$, Béla Ozsvári ${ }^{1,2}$, Maria Peiris-Pagès ${ }^{1,2}$, Marco \\ Fiorillo $^{1,2,3}$, Duncan L. Smith ${ }^{4}$, Generoso Bevilacqua ${ }^{5,6}$, Chiara Maria Mazzanti ${ }^{5}$, \\ Liam A. McDonnell5, Antonio Giuseppe Naccarato6, Maybo Chiu ${ }^{1,2}$, Luke Wynne ${ }^{1,2}$, \\ Ubaldo E. Martinez-Outschoorn7, Federica Sotgia ${ }^{1,2}$ and Michael P. Lisanti, ${ }^{1,2}$ \\ ${ }^{1}$ The Breast Cancer Now Research Unit, Institute of Cancer Sciences, University of Manchester, Manchester, UK \\ 2 The Manchester Centre for Cellular Metabolism (MCCM), Institute of Cancer Sciences, University of Manchester, Manchester, \\ UK \\ ${ }^{3}$ The Department of Pharmacy, Health and Nutritional Sciences, The University of Calabria, Cosenza, Italy \\ ${ }^{4}$ The Cancer Research UK Manchester Institute, University of Manchester, Manchester, UK \\ ${ }^{5}$ FPS - The Pisa Science Foundation, Pisa, Italy \\ ${ }^{6}$ Department of Pathology, Pisa University Hospital, Pisa, Italy \\ 7 The Sidney Kimmel Cancer Center, Philadelphia, PA, USA \\ * These authors should be considered as co-first authors
}

Correspondence to: Michael P. Lisanti, email: michaelp.lisanti@gmail.com

Federica Sotgia, email: fsotgia@gmail.com

Keywords: mitochondria, MitoTracker, MMTV, WNT, FGF

Received: July 21,2015 Accepted: August 22, $2015 \quad$ Published: September 28, 2015

This is an open-access article distributed under the terms of the Creative Commons Attribution License, which permits unrestricted use, distribution, and reproduction in any medium, provided the original author and source are credited.

\section{ABSTRACT}

Here, we developed an isogenic cell model of "stemness" to facilitate protein biomarker discovery in breast cancer. For this purpose, we used knowledge gained previously from the study of the mouse mammary tumor virus (MMTV). MMTV initiates mammary tumorigenesis in mice by promoter insertion adjacent to two main integration sites, namely Int-1 (Wnt1) and Int-2 (Fgf3), which ultimately activates $W n t / \beta$-catenin signaling, driving the propagation of mammary cancer stem cells (CSCs). Thus, to develop a humanized model of MMTV signaling, we overexpressed WNT1 and FGF3 in MCF7 cells, an ER( + ) human breast cancer cell line. We then validated that MCF7 cells over-expressing both WNT1 and FGF3 show a 3.5fold increase in mammosphere formation, and that conditioned media from these cells is also sufficient to promote stem cell activity in untransfected parental MCF7 and T47D cells, as WNT1 and FGF3 are secreted factors. Proteomic analysis of this model system revealed the induction of $\mathrm{i}$ ) EMT markers, ii) mitochondrial proteins, iii) glycolytic enzymes and iv) protein synthesis machinery, consistent with an anabolic CSC phenotype. MitoTracker staining validated the expected WNT1/FGF3-induced increase in mitochondrial mass and activity, which presumably reflects increased mitochondrial biogenesis. Importantly, many of the proteins that were up-regulated by WNT/FGF-signaling in MCF7 cells, were also transcriptionally over-expressed in human breast cancer cells in vivo, based on the bioinformatic analysis of public gene expression datasets of laser-captured patient samples. As such, this isogenic cell model should accelerate the discovery of new biomarkers to predict clinical outcome in breast cancer, facilitating the development of personalized medicine.

Finally, we used mitochondrial mass as a surrogate marker for increased mitochondrial biogenesis in untransfected MCF7 cells. As predicted, metabolic 
fractionation of parental MCF7 cells, via MitoTracker staining, indicated that high mitochondrial mass is a new metabolic biomarker for the enrichment of anabolic CSCs, as functionally assessed by mammosphere-forming activity. This observation has broad implications for understanding the role of mitochondrial biogenesis in the propagation of stem-like cancer cells. Technically, this general metabolic approach could be applied to any cancer type, to identify and target the mitochondrial-rich CSC population.

The implications of our work for understanding the role of mitochondrial metabolism in viral oncogenesis driven by random promoter insertions are also discussed, in the context of MMTV and ALV infections.

\section{INTRODUCTION}

The mouse mammary tumor virus (MMTV) is a saliva- and milk-transmitted retrovirus [1-5]; however, infected mice only develop mammary tumors in adulthood [4]. This long latency period makes MMTV an interesting virus for understanding the pathogenesis of human breast cancers [6]. The provirus inserts upstream of two key integration sites, named Int-1 and Int-2 [7-10]. This process of insertional mutatgenesis is thought to be random, but involves the positive selection of genes that will ultimately provide an increase in "stemness", a cellular growth-advantage, or perhaps both. MMTV tumors are oligo-clonal, suggesting that there is some synergy between these two different integration sites. These mammary proto-oncogenes Int- 1 and Int- 2 have been identified as WNT1 and FGF3 [11-13], two secreted growth factors normally involved in stem cell signaling pathways.

WNT1 is the first member of the WNT gene family, which is known to be involved in cell fate determination and patterning during embryogenesis $[14,15]$. FGF3 is a member of the fibroblast growth factor family, which controls cell proliferation, morphogenesis and tissue repair [16]. Interestingly, WNT1 and FGF3 converge directly upon the WNT/ $\beta$-catenin signaling cascade [17, 18]. However, it remains largely unknown exactly how WNT1/FGF3 signaling induces mammary tumorigenesis.

Here, we have created a humanized model of MMTV signaling, by over-expressing WNT1 and FGF3 in human breast cancer cells, namely MCF7 cells, an $\mathrm{ER}(+)$ cell line. Unbiased label-free proteomic analysis of this model system reveals the induction of EMT markers, mitochondrial proteins, glycolytic enzymes and protein synthesis machinery, consistent with an anabolic CSC phenotype. The proteins that were up-regulated by WNT/ FGF-signaling in MCF7 cells, were also transcriptionally over-expressed in human breast cancer cells in vivo. This isogenic cell model should accelerate the identification and development of new protein biomarkers to predict clinical outcomes in breast cancer patients.

Finally, we also show that mitochondrial mass is a new metabolic biomarker for anabolic CSCs, as assessed by MitoTracker vital-staining and metabolic cell fractionation by flow-cytometry.

\section{RESULTS}

Generating a humanized model of MMTV signaling.

During MMTV infection of mammary epithelial cells, genomic viral integration occurs. This ultimately leads to mammary tumorigenesis in mice. Mechanistically,

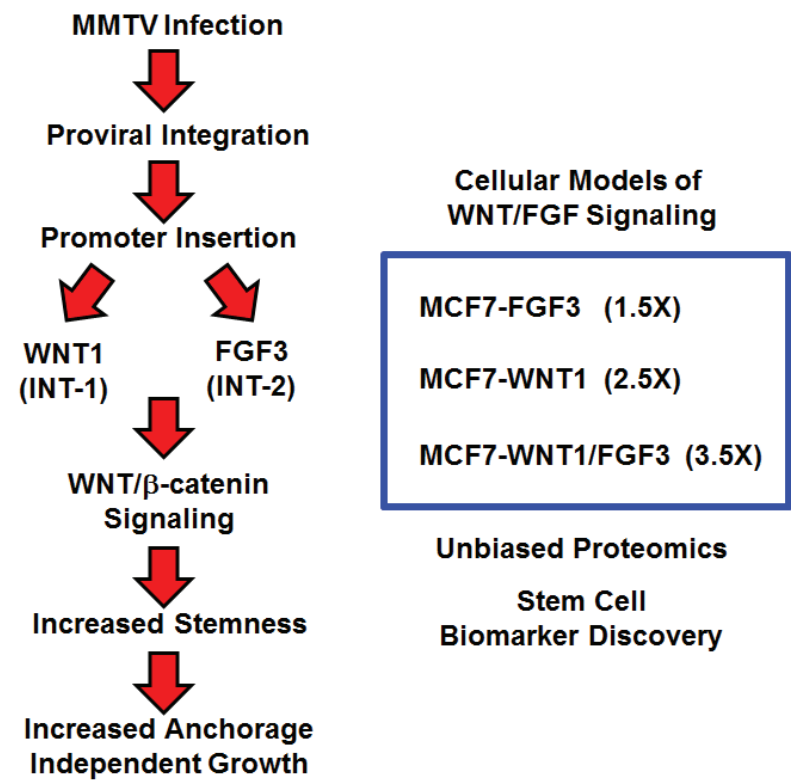

Figure 1: Creating a humanized experimental model for MMTV: Focus on WNT1 and FGF3 signaling. To create a humanized model of MMTV signaling, we recombinantly over-expressed WNT1 and FGF3 in MCF7 cells, an $\mathrm{ER}(+)$ human breast cancer cell line. WNT1 and FGF3 were expressed either individually or in combination, using lenti-viral vectors carrying two different selection markers (puromycin or neomycin/G418). This isogenic cell model of "stemness" was generated to facilitate protein biomarker discovery in breast cancer, via unbiased label-free proteomics. Importantly, over-expression of FGF3, WNT1, or WNT1/FGF3 increases mammosphere formation by 1.5-, 2.5 - and 3.5 -fold, respectively (See Figure 2). Thus, we focused on MCF7-WNT1/FGF3 cells for further validation and proteomic analysis. 
Table 1: MMTV common proviral integration sites and gene designations.

$\begin{array}{ll}\text { Integration site(s) } & \text { Gene Name } \\ \text { Int-1 } & \text { Wnt-1 } \\ \text { Int-2 } & \text { Fgf-3 } \\ \text { Int-3 } & \text { Notch-4 } \\ \text { Int-4 } & \text { Wnt-3 } \\ \text { Int-5 } & \text { Aromatase; Cyp19a1 } \\ \text { Int-6 } & \text { Eukaryotic translation initiation factor 3; elF3 } \\ \text { Int-7 } & \text { Rspo-2 }\end{array}$

Additional genes include Fgf-4, Wnt-3a and Wnt-10, among others.

the MMTV virus uses a promoter insertion mechanism of mutagenesis, to drive oncogenesis [19]. More specifically, the MMTV promoter inserts upstream of two main integration sites, namely Int-1 (Wnt1) or Int-2 (Fgf3), although a few other rare integration sites have been described [11-13, 20] (Table 1). As a consequence, the MMTV promoter drives the over-expression of these secreted stem cell associated growth factors, constitutively activating $\mathrm{Wnt} / \beta$-catenin signaling $[11,21,22]$. Thus, the MMTV model has been instrumental for understanding how "amplified" or "constitutive" stem cell signaling directly contributes to solid tumor formation (Figure 1). As such, it would be beneficial to create a humanized non-infectious model of MMTV signaling, to drive the discovery of new stem cell associated biomarkers, for predicting clinical outcome in human breast cancer

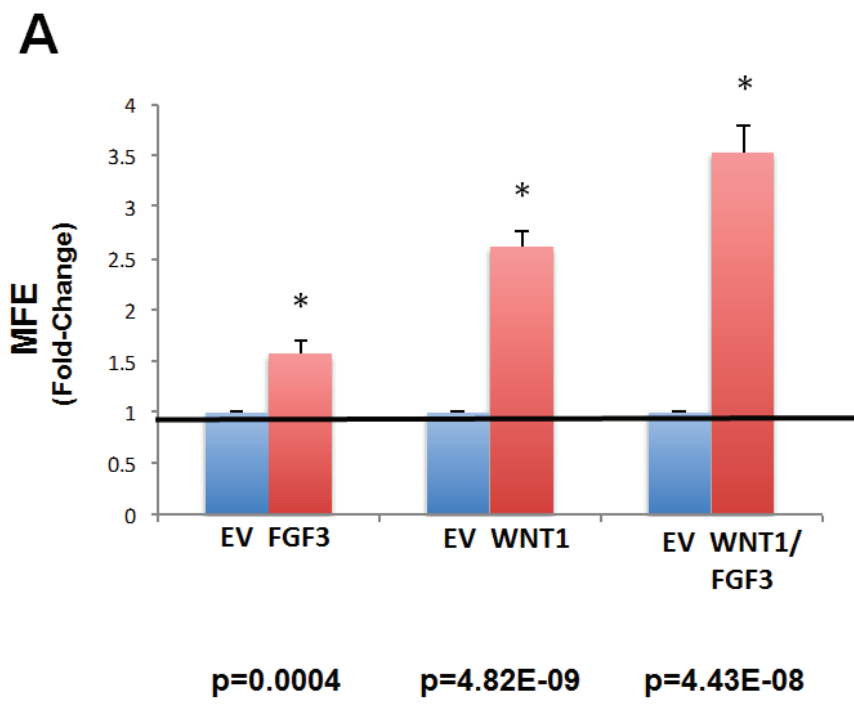

patients.

Thus, in order to create a humanized model of MMTV signaling, we recombinantly over-expressed WNT1 and FGF3 in MCF7 cells, an ER(+) human breast cancer cell line. WNT1 and FGF3 were expressed either individually or in combination, using lentiviral vectors carrying two different selection markers (puromycin or neomycin/G418). For comparison purposes, empty vector controls (EV) were generated in parallel (Figure 1).

Then, the various cell lines were screened for stem cell activity, using the mammosphere assay as a functional readout. Importantly, Figure 2A directly validates that over-expression of either WNT1 or FGF3 is sufficient to increase the clonal expansion of cancer stem cells. Interestingly, FGF3 and WNT1 significantly increased mammosphere formation by $\sim 1.5$-fold and $\sim 2.5$-fold,

B

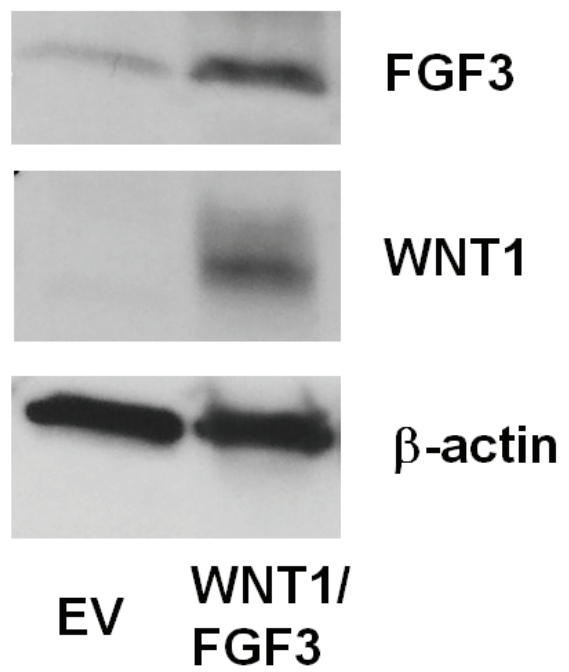

Figure 2: Recombinant over-expression of WNT1 and/or FGF3 in MCF7 cells significantly augments mammosphere formation. A) Mammosphere formation. The cell lines we generated were screened for stem cell activity, using the mammosphere assay as a functional readout. Note that over-expression of either WNT1 or FGF3 significantly increased mammosphere formation by $\sim 1.5$-fold and $\sim 2.5$-fold, respectively. However, MCF7 cells over-expressing both WNT1 and FGF3 showed the largest increase in mammosphere formation, by up to $\sim 3.5$-fold. Results were normalized to the three control cell lines harboring either i) EX-Neg-Lv105(puro), ii) EXNeg-Lv151(neo) or iii) both control vectors. P-values are as indicated. Assays were performed in triplicate and repeated three times independently. MFE, mammosphere forming efficiency. B) Immunoblot analysis. Recombinant over-expression of WNT1 and FGF3 in these transfected cell models was validated by immunoblot analysis, with specific antibody probes. Beta-actin is shown as a control for equal loading. 
respectively. However, MCF7 cells over-expressing both WNT1 and FGF3 showed the largest increase in mammosphere formation, by up to $\sim 3.5$-fold (Figure 2A).

We also validated the recombinant over-expression of WNT1 and FGF3 in these transfected cell models by immunoblot analysis, with specific antibody probes (Figure 2B).

Conditioned media from MCF7-WNT1/FGF3 cells is sufficient to increase mammosphere formation.

As WNT1 and FGF3 are secreted factors, it would be predicted that the increase in constitutive stem cell signaling could also act in a paracrine fashion on nontransfected cells. To test this hypothesis, we prepared conditioned media from MCF7-WNT1/FGF3 cells and the corresponding empty vector (MCF7-EV) control cells.

Then, we compared the ability of conditioned media to support mammosphere formation, in untransfected parental MCF7 cells. Figure 3 (Left) shows that conditioned media prepared from MCF7-WNT1/FGF3 cells significantly stimulated mammosphere formation by $\sim 2$-fold. Importantly, virtually identical results were obtained with untransfected parental T47D cells, a second independent $\operatorname{ER}(+)$ breast cancer cell line (Figure 3
(Right)).

Proteomics analysis of MCF7-WNT1/FGF3 cells reveals the upregulation of EMT markers, mitochondrial proteins, glycolytic enzymes, and the protein synthesis machinery.

To better understand how WNT and FGF signaling drive the expansion of CSCs, we used unbiased label-free proteomics analysis. The proteome of MCF7-WNT1/ FGF3 cells was compared to MCF7-EV control cells. We restricted our analysis to protein products that were overexpressed by $>1.5$-fold. Overall, our results are detailed in Tables 2, 3 and 4 .

Remarkably, $>40$ nuclear-encoded mitochondrialrelated proteins were over-expressed in MCF7-WNT1/ FGF3 cells (Table 2). Many of these proteins were related to the TCA cycle (ACO2), oxidative phosphorylation (MT$\mathrm{CO} 2)$, regenerating ATP (CKMT1/2) or mitochondrial biogenesis (TOMM34). In addition, MT-CO2 (a mitochondria-DNA encoded protein) was upregulated by $>2.5$-fold, indicative of the production of new mitochondria. In support of an anabolic phenotype, proteins related to glycolysis, the pentose phosphate pathway, glycogen metabolism and amino acid synthesis were upregulated (Table 2).

\section{WNT1/FGF3 Conditioned Media Experiments}

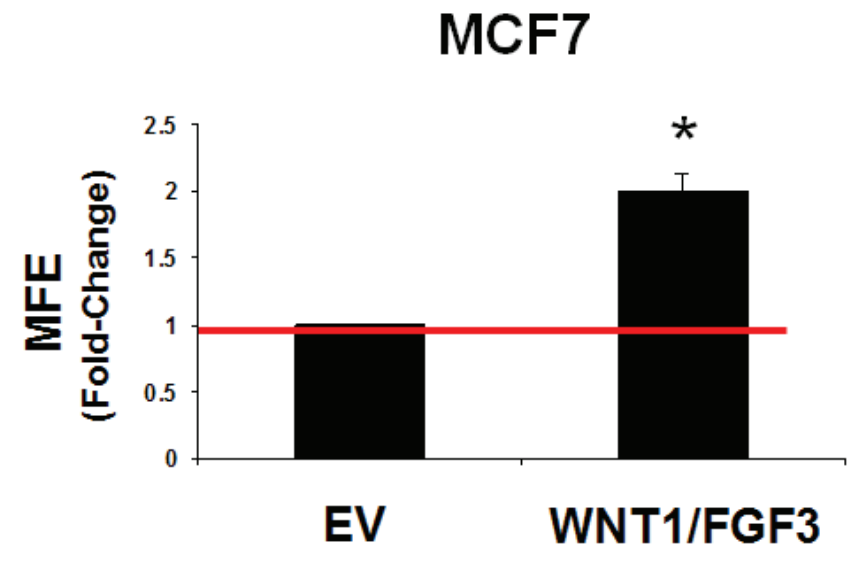

$\mathrm{p}=1.11 \mathrm{E}-06$

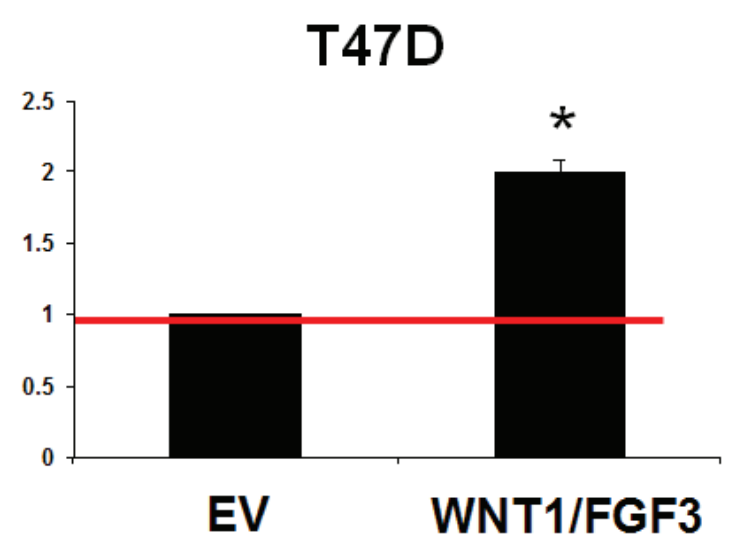

$\mathrm{p}=1.58 \mathrm{E}-08$

Figure 3: Conditioned media from WNT1/FGF3 expressing MCF7 cells increases mammosphere formation. Since WNT1 and FGF3 are secreted factors, they should act in a paracrine fashion on non-transfected cells. To test this hypothesis, we prepared conditioned media from MCF7-WNT1/FGF3 cells and the corresponding empty vector (MCF7-EV) control cells. Then, we compared the ability of this conditioned media to support mammosphere formation, in untransfected parental MCF7 cells (Left panel). Note that conditioned media prepared from MCF7-WNT1/FGF3 cells significantly stimulated mammosphere formation by $\sim 2$-fold. Nearly identical results were obtained with untransfected parental T47D cells, a second independent ER(+) breast cancer line (Right panel). Assays were performed in triplicate and repeated three times independently. MFE, mammosphere forming efficiency. 
Table 2: Key Molecules Up-regulated by WNT1/FGF3 in MCF7 Cells: Mitochondria and Glycolysis.

\begin{tabular}{|c|c|c|c|}
\hline Symbol & Description & Fold-Change & ANOVA \\
\hline \multicolumn{4}{|c|}{ Mitochondrial-related Proteins/TCA Cycle (42) } \\
\hline$\overline{\mathrm{ACO} 2}$ & Aconitase 2, mitochondrial & Infinity & $1.61 \mathrm{E}-08$ \\
\hline IDH1 & Isocitrate dehydrogenase [NADP] 1 & Infinity & 0.004 \\
\hline $\mathrm{MDH} 1$ & Malate dehydrogenase, cytoplasmic & 28.32 & 2.11E-05 \\
\hline CKMT2 & Creatine kinase S-type, mitochondrial & 11.04 & 0.015 \\
\hline FASN & Fatty acid synthase & 7.77 & 0.036 \\
\hline CKMT1 & Creatine kinase U-type, mitochondrial & 7.60 & 2.12E-05 \\
\hline CKMT1B & Creatine kinase U-type, mitochondrial & 6.27 & $5.74 \mathrm{E}-05$ \\
\hline CKMT1A & Creatine kinase U-type, mitochondrial & 5.19 & 7.98E-05 \\
\hline OAT & Ornithine aminotransferase, mitochondrial & 4.79 & 0.0003 \\
\hline $\mathrm{PC}$ & Pyruvate carboxylase, mitochondrial & 4.63 & 0.0001 \\
\hline DUT & Deoxyuridine 5'-triphosphate nucleotidohydrolase, mitochondrial & 3.85 & 0.0001 \\
\hline TOMM34 & Translocase of outer mitochondrial membrane 34 & 2.89 & 0.0027 \\
\hline GLUD2 & Glutamate dehydrogenase 2, mitochondrial & 2.78 & 0.035 \\
\hline MT-CO2 & Cytochrome c oxidase subunit 2 (COX2) & 2.66 & 0.0017 \\
\hline NQO1 & $\mathrm{NAD}(\mathrm{P}) \mathrm{H}$ dehydrogenase [quinone] 1 & 2.60 & 8.31E-05 \\
\hline ACADVL & Very long-chain-specific acyl-CoA dehydrogenase, mitochondrial & 2.46 & 0.0007 \\
\hline C21orf33 & ES1 protein homolog, mitochondrial & 2.37 & 0.01 \\
\hline NDUFS1 & Mitochondrial NADH-ubiquinone oxidoreductase 75 kDa subunit & 2.27 & 0.0026 \\
\hline SCD & Acyl-CoA desaturase & 2.25 & 3.93E-05 \\
\hline GPD2 & Glycerol-3-phosphate dehydrogenase, mitochondrial & 2.08 & 0.03 \\
\hline HSPA9 & Stress-70 protein, mitochondrial & 2.07 & 0.0498 \\
\hline IDH3A & Isocitrate dehydrogenase [NAD] subunit alpha, mitochondrial & 1.96 & 0.001 \\
\hline HSPD1 & $60 \mathrm{kDa}$ heat shock protein, mitochondrial & 1.95 & 0.02 \\
\hline ETFA & Electron transfer flavoprotein subunit alpha, mitochondrial & 1.91 & 0.03 \\
\hline ABAT & 4-aminobutyrate aminotransferase, mitochondrial & 1.85 & 0.03 \\
\hline PRDX5 & Peroxiredoxin-5, mitochondrial & 1.83 & 0.037 \\
\hline $\mathrm{COX} 411$ & Cytochrome c oxidase subunit 4 isoform 1 , mitochondrial & 1.83 & 0.007 \\
\hline COX6A1 & Cytochrome c oxidase subunit $6 \mathrm{~A}$, mitochondrial & 1.81 & 0.002 \\
\hline TUFM & Elongation factor Tu, mitochondrial & 1.78 & 0.02 \\
\hline ATP50 & ATP synthase subunit $O$, mitochondrial & 1.77 & 0.004 \\
\hline CLPX & ATP-dependent Clp protease ATP-binding subunit clpX-like, mitochondrial & 1.72 & 0.016 \\
\hline CS & Citrate synthase, mitochondrial & 1.71 & 0.001 \\
\hline ECHS1 & Enoyl-CoA hydratase, mitochondrial & 1.70 & 0.004 \\
\hline ATP5B & ATP synthase subunit beta, mitochondrial & 1.69 & 0.039 \\
\hline PCK2 & Phosphoenolpyruvate carboxykinase [GTP], mitochondrial & 1.66 & 0.001 \\
\hline AK2 & Adenylate kinase 2 , mitochondrial & 1.65 & 0.004 \\
\hline ATP5A1 & ATP synthase subunit alpha, mitochondrial & 1.61 & 0.02 \\
\hline ETFB & Electron-transfer-flavoprotein, beta & 1.60 & 0.02 \\
\hline PRKDC & DNA-dependent protein kinase catalytic subunit (maintains mt-DNA copy number) & 1.58 & 0.03 \\
\hline $\mathrm{CHCHD2P9}$ & Coiled-coil-helix-coiled-coil-helix domain-protein $\mathrm{CHCHD} 2 \mathrm{P9}$, mitochondrial & 1.57 & 0.02 \\
\hline AIFM1 & Apoptosis-inducing factor 1 , mitochondrial & 1.53 & 0.006 \\
\hline UQCRFS1P1 & Putative cytochrome b-c1 complex subunit Rieske-like protein 1 & 1.50 & 0.02 \\
\hline \multicolumn{4}{|c|}{ Enzymes Related to Glycolysis, the Pentose Phosphate Pathway, Glycogen, and Amino Acid Synthesis (Serine/Arginine) (14) } \\
\hline PHGDH & D-3-phosphoglycerate dehydrogenase & Infinity & 2.68E-13 \\
\hline ASS1 & Argininosuccinate synthase & 17.39 & $3.42 E-09$ \\
\hline HK2 & Hexokinase-2 & 11.57 & $3.15 \mathrm{E}-08$ \\
\hline PKM2 & Pyruvate kinase & 2.83 & 0.003 \\
\hline PYGB & Glycogen phosphorylase, brain form & 2.23 & 0.01 \\
\hline PFKL & 6-phosphofructokinase, liver type & 2.17 & 0.004 \\
\hline CAD & Glutamine-dependent carbamoyl-phosphate synthase & 2.08 & 0.0007 \\
\hline PKLR & Pyruvate kinase isozymes $R / L$ & 2.08 & 1.46E-05 \\
\hline PGAM4 & Probable phosphoglycerate mutase 4 & 1.94 & 0.005 \\
\hline G6PD & Glucose-6-phosphate 1-dehydrogenase & 1.89 & 0.005 \\
\hline TKT & Transketolase & 1.64 & 0.003 \\
\hline PGK2 & Phosphoglycerate kinase 2 & 1.57 & 0.006 \\
\hline ENO1 & Enolase, alpha & 1.51 & 0.007 \\
\hline PGK1 & Phosphoglycerate kinase & 1.50 & 0.04 \\
\hline
\end{tabular}

Stem-like cancer cells also undergo an epithelialmesenchymal transition (EMT), which promotes cell migration, invasion and distant metastasis [23]. Importantly, $>45$ proteins known to be associated with the EMT phenotype (cell migration or invasiveness) were upregulated in MCF7-WNT1/FGF3 cells (Table 3).
Examples include FRS2 (FGF receptor substrate-2; $>10$ fold) and $\beta$-catenin ( $>2$-fold), which would be expected to further amplify WNT/FGF signaling, as these are downstream elements of these convergent signaling networks. Similarly, other signaling molecules that promote the EMT and cell migration were significantly upregulated, such as 
Table 3: Key Molecules Up-requlated by WNT1/FGF3 in MCF7 Cells: The EMT and Cell Migration.

\begin{tabular}{|c|c|c|c|}
\hline Symbol & Description & Fold-Change & ANOVA \\
\hline \multicolumn{4}{|c|}{ EMT Markers, Extracellular Matrix, Cell Migration and Cytoskeletal proteins (47) } \\
\hline MARCKS & Myristoylated alanine-rich C-kinase substrate & 371.76 & $3.72 \mathrm{E}-05$ \\
\hline S100A14 & Protein S100-A14 & 96.23 & 0.0002 \\
\hline CDC42 & Cell division control protein 42 homolog & 68.67 & 5.27E-06 \\
\hline LGALS3BP & Galectin-3-binding protein & 38.46 & 0.001 \\
\hline FRS2 & Fibroblast growth factor receptor substrate 2 & 11.59 & 9.69E-05 \\
\hline MAST4 & Microtubule-associated serine/threonine-protein kinase 4 & 10.81 & $2.62 \mathrm{E}-05$ \\
\hline CALML5 & Calmodulin-like protein 5 & 8.06 & 0.0007 \\
\hline CDV3 & Carnitine deficiency-associated gene expressed in cardiac ventricle 3 & 7.67 & $7.58 \mathrm{E}-06$ \\
\hline SCUBE1 & Signal peptide, CUB domain, EGF-like 1 & 6.84 & 1.97E-05 \\
\hline S100A11 & Protein S100-A11 & 6.66 & 0.026 \\
\hline S100A16 & Protein S100-A16 & 6.41 & 0.0003 \\
\hline MERTK & Tyrosine-protein kinase MER & 6.24 & 4.40E-05 \\
\hline NINJ1 & Ninjurin-1 & 5.36 & 0.0001 \\
\hline TTBK2 & Tau-tubulin kinase & 5.31 & 4.15E-05 \\
\hline EMD & Emerin & 4.38 & 0.0006 \\
\hline FLNB & Filamin-B & 4.27 & 0.004 \\
\hline TTN & Titin & 3.66 & 7.32E-05 \\
\hline CGNL1 & Cingulin-like protein 1 & 3.62 & 0.005 \\
\hline TAGLN2 & Transgelin-2 & 2.94 & 0.02 \\
\hline ACTA2 & Actin, aortic smooth muscle & 2.86 & 0.0002 \\
\hline TLN1 & Talin-1 & 2.78 & 0.008 \\
\hline SEPT2 & Septin-2 & 2.77 & 0.004 \\
\hline HMGB1 & High mobility group protein B1 & 2.66 & 0.001 \\
\hline TPT1 & Translationally-controlled tumor protein & 2.54 & 0.028 \\
\hline AMOT & Angiomotin & 2.40 & 0.008 \\
\hline CTNNB1 & Catenin, beta-1 & 2.37 & 0.0003 \\
\hline $\begin{array}{l}\text { TRIOBP } \\
\text { ASAP2 }\end{array}$ & $\begin{array}{l}\text { TRIO and F-actin-binding protein } \\
\text { Arf-GAP with SH3 domain, ANK repeat and } \mathrm{PH} \text { domain-containing protein } 2\end{array}$ & $\begin{array}{l}2.35 \\
2.23\end{array}$ & $\begin{array}{l}0.003 \\
0.01\end{array}$ \\
\hline MYH14 & Myosin-14 & 2.20 & 0.02 \\
\hline S100A10 & S100A10 protein & 2.13 & 0.0002 \\
\hline TAX1BP3 & Tax1-binding protein 3 & 2.13 & 0.0002 \\
\hline HMGB3 & High mobility group protein B3 & 2.10 & 0.01 \\
\hline FLNA & Filamin-A & 2.08 & 0.004 \\
\hline MYO18B & Myosin XVIIIB & 1.99 & 0.0005 \\
\hline IQGAP1 & IQ motif containing GTPase activating protein 1 (scaffold protein for CDC42) & 1.98 & 0.026 \\
\hline ACTN2 & Alpha-actinin-2 & 1.96 & 1.64E-05 \\
\hline ANXA2 & Annexin A2 & 1.90 & 0.01 \\
\hline TAGLN3 & Transgelin-3 & 1.89 & 0.01 \\
\hline FAM129B & Niban-like protein 1 (associated with cell invasion) & 1.88 & 0.0008 \\
\hline ACTN3 & Alpha-actinin-3 & 1.81 & 0.025 \\
\hline FAM82B & Regulator of microtubule dynamics protein 1 & 1.80 & 0.02 \\
\hline MYH10 & Myosin, heavy polypeptide 10, non-muscle & 1.79 & 0.04 \\
\hline MYOF & Myoferlin & 1.60 & 0.0026 \\
\hline CAPZB & F-actin-capping protein subunit beta & 1.58 & 0.04 \\
\hline MTPN & Myotrophin & 1.57 & 0.007 \\
\hline TUBB2A & Tubulin beta-2A chain & 1.56 & 0.0045 \\
\hline EPPK1 & Epiplakin & 1.51 & 0.0485 \\
\hline \multicolumn{4}{|c|}{ Miscellaneous (17) } \\
\hline CAST & Calpastatin A & 21.60 & 0.005 \\
\hline SH3BGRL & SH3 domain-binding glutamic acid-rich-like protein & 14.23 & 0.0003 \\
\hline SEC24A & Protein transport protein Sec24A & 12.52 & 7.39E-05 \\
\hline PABPC4 & Polyadenylate-binding protein 4 & 6.53 & 0.0006 \\
\hline C10orf12 & Uncharacterized protein C10orf12 & 5.71 & 3.46E-05 \\
\hline TMED4 & Transmembrane emp24 domain-containing protein 4 & 4.99 & 4.34E-05 \\
\hline PTMS & Parathymosin & 4.66 & 0.0001 \\
\hline HUWE1 & E3 ubiquitin-protein ligase HUWE1 & 4.50 & 5.23E-05 \\
\hline PON2 & Paraoxonase 2 , isoform & 4.28 & 0.003 \\
\hline $\begin{array}{l}\text { AHNAK } \\
\text { COMT }\end{array}$ & $\begin{array}{l}\text { Neuroblast differentiation-associated protein, AHNAK } \\
\text { Soluble catechol-O-methyltransferase }\end{array}$ & $\begin{array}{l}3.68 \\
3.66\end{array}$ & 0.0002 \\
\hline STUB1 & E3 ubiquitin-protein ligase CHIP & 3.38 & 0.0001 \\
\hline TMEM205 & Transmembrane protein 205 (chemo-resistance to cisplatin) & 2.92 & $1.55 \mathrm{E}-05$ \\
\hline TFF1 & Trefoil factor 1 & 2.30 & 0.005 \\
\hline MATR3 & Matrin-3 & 2.25 & 0.038 \\
\hline SRRM2 & Serine/arginine repetitive matrix protein 2 & 2.19 & 0.03 \\
\hline ARF5 & ADP-ribosylation factor 5 & 2.06 & 0.009 \\
\hline
\end{tabular}

Genes encoding FRS2 and CTNNB1 are highlighted in BOLD, as they would be expected to amplify FGF and WNT signaling, respectively. 
Table 4: Key Molecules Up-regulated by WNT1/FGF3 in MCF7 Cells: Ribosomes and Protein Synthesis.

\begin{tabular}{|c|c|c|c|}
\hline Symbol & Description & Fold-Change & ANOVA \\
\hline \multicolumn{4}{|c|}{ Ribosome-related proteins (8) } \\
\hline RPL13 & $60 S$ ribosomal protein L13 & 6.65 & 7.43E-07 \\
\hline NPM1 & NPM1 protein & 3.78 & 0.001 \\
\hline RPL14 & 60 S ribosomal protein L14 & 3.41 & 0.001 \\
\hline SRPRB & Signal recognition particle receptor subunit beta & 2.11 & 0.002 \\
\hline RPL4 & 60 S ribosomal protein $\mathrm{L} 4$ & 2.04 & 0.01 \\
\hline RPS5 & $40 S$ ribosomal protein S5 & 1.99 & 0.006 \\
\hline RPL15 & $60 S$ ribosomal protein $\mathrm{L} 15$ & 1.99 & 0.004 \\
\hline RPL13 & 60S ribosomal protein L19 & 1.50 & 0.01 \\
\hline \multicolumn{4}{|c|}{ Translation initiation factors $(5)$} \\
\hline EIF5A & Eukaryotic translation initiation factor $5 \mathrm{~A}$ & 7.22 & 0.03 \\
\hline EIF5B & Eukaryotic translation initiation factor $5 \mathrm{~B}$ & 2.25 & 3.38E-06 \\
\hline EIF6 & Eukaryotic translation initiation factor 6 & 2.24 & 0.028 \\
\hline EIF2S1 & Eukaryotic translation initiation factor 2 , subunit 1 alpha, $35 \mathrm{kDa}$ & 1.55 & 0.0035 \\
\hline EIF3D & Eukaryotic translation initiation factor 3 subunit $D$ & 1.51 & 0.03 \\
\hline \multicolumn{4}{|c|}{ Elongation factors (4) } \\
\hline EEF1B2 & Elongation factor 1-beta & 1.95 & 0.0498 \\
\hline TUFM & Elongation factor Tu, mitochondrial & 1.78 & 0.02 \\
\hline EEF1D & Elongation factor 1-delta & 1.70 & 0.03 \\
\hline EEF1G & Elongation factor 1-gamma & 1.52 & 0.001 \\
\hline \multicolumn{4}{|c|}{ Enzymes for tRNA synthesis (6) } \\
\hline DARS & Aspartate--tRNA ligase, cytoplasmic & 3.23 & 0.0001 \\
\hline WARS & Tryptophan--tRNA ligase, cytoplasmic & 2.54 & 0.0016 \\
\hline LARS & Leucine--tRNA ligase, cytoplasmic & 1.90 & 0.002 \\
\hline FARSB & Phenylalanine--tRNA ligase beta subunit & 1.69 & 0.005 \\
\hline EPRS & Bifunctional aminoacyl-tRNA synthetase (Glutamyl-Prolyl-tRNA Synthetase) & 1.65 & 0.006 \\
\hline C22orf28 & tRNA-splicing ligase RtcB homolog & 1.56 & 0.008 \\
\hline \multicolumn{4}{|c|}{ Protein folding chaperones (heat shock proteins) (14) } \\
\hline$\overline{P D I A 3}$ & Protein disulfide-isomerase $\mathrm{A} 3$ & 3.59 & 2.39E-05 \\
\hline PPIB & Peptidyl-prolyl cis-trans isomerase B & 3.39 & $1.90 \mathrm{E}-06$ \\
\hline CALU & Calumenin & 2.93 & 0.0002 \\
\hline PDIA6 & Protein disulfide-isomerase $\mathrm{A} 6$ & 2.82 & 0.001 \\
\hline PDIA4 & Protein disulfide-isomerase A4 & 2.68 & 0.004 \\
\hline HSPA1B & Heat shock $70 \mathrm{kDa}$ protein 1 & 2.43 & 0.0003 \\
\hline HSPD1 & $60 \mathrm{kDa}$ heat shock protein, mitochondrial & 1.95 & 0.02 \\
\hline HSP90AB3P & Putative heat shock protein HSP 90-beta-3 & 1.61 & 0.026 \\
\hline HSPA8 & Heat shock cognate $71 \mathrm{kDa}$ protein & 1.60 & 0.02 \\
\hline HSP90B1 & Endoplasmin & 1.60 & 0.006 \\
\hline HSPH1 & Heat shock protein $105 \mathrm{kDa}$ & 1.60 & 0.047 \\
\hline PPIA & Peptidyl-prolyl cis-trans isomerase A & 1.60 & 0.02 \\
\hline HSP90AB1 & Heat shock protein HSP 90-beta & 1.57 & 0.049 \\
\hline CANX & Calnexin & 1.54 & 0.01 \\
\hline \multicolumn{4}{|c|}{ Amino acid transport (2) } \\
\hline SLC1A5 & Neutral amino acid transporter $\mathrm{B}(0)$ & 2.77 & 0.0004 \\
\hline SLC7A5 & Solute carrier family 7 (Cationic amino acid transporter, y+ system), member 5 & 1.66 & 0.04 \\
\hline
\end{tabular}

MARCKS ( $>370$-fold) and CDC42 ( $>65$-fold).

Finally, augmented protein synthesis is another characteristic of anabolic CSCs. Note that MCF7-WNT1/ FGF3 cells show the upregulation of $>35$ proteins related to protein synthesis (Table 4). Examples include ribosomerelated proteins (RPS and RPL), translation initiation factors (EIFs), peptide elongation factors (EEFs), enzymes for tRNA synthesis, as well as chaperones for protein folding (HSPs) and amino acid transporters (SLC).

Thus, MCF7-WNT1/FGF3 cells upregulate greater than 140 proteins that would be consistent with an overall anabolic phenotype (Figure 4).

\section{Expression of WNT1/FGF3-related targets in patient-derived human breast cancer samples}

To determine the possible translational significance of our results, we intersected our WNT-FGF proteomics data with human genome-wide transcriptional profiling data. These human clinical data were derived from publically available human breast cancer samples, in which breast cancer cells were separated by laser-capture microdissection from tumor stromal cells. Transcriptional profiles were analyzed from from $\mathrm{N}=28$ human breast cancer patients (See the Materials \& Methods section). In this data set, gene expression was previously determined 
using Affymetrix U133A 2.0 GeneChips. A concise summary of these findings is presented in Tables 5, 6 and 7. Overall, greater than sixty WNT1/FGF3 targets (related to mitochondria, glycolysis, the EMT, and protein synthesis) that we identified in MCF7-WNT1/FGF3 cells were also transcriptionally elevated in human breast cancer cells in vivo. These new protein targets that we identified in MCF7-WNT1/FGF3 cells may be important for developing new strategies for the diagnosis and treatment of breast cancer.

\section{Anabolic CSC Phenotype}

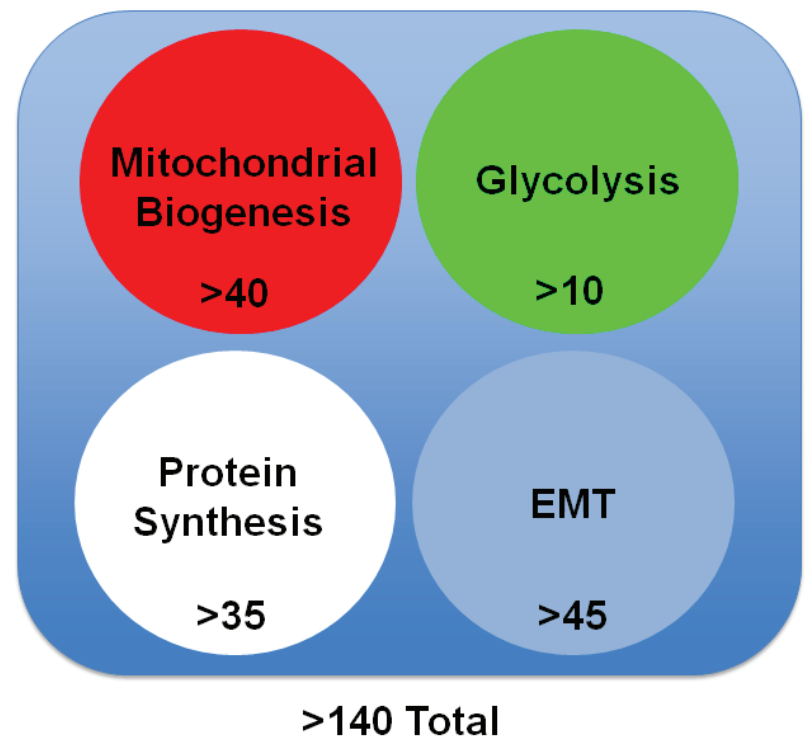

Figure 4: The anabolic CSC phenotype: Proteomics analysis. Unbiased label-free proteomics analysis of MCF7WNT1/FGF3 cells revealed the induction of i) mitochondrial proteins, ii) glycolytic enzymes, iii) protein synthesis machinery and iv) EMT markers, consistent with an anabolic CSC phenotype. For specific details, see Tables 2, 3 and 4. Mitochondrial proteins - Greater than 40 nuclear-encoded mitochondrial-related proteins were over-expressed in MCF7WNT1/FGF3 cells. Many of these proteins were related to the TCA cycle (ACO2), oxidative phosphorylation (MT-CO2), regenerating ATP (CKMT1/2) or mitochondrial biogenesis (TOMM34). In addition, MT-CO2 (a mitochondrial DNA encoded protein) was upregulated by $>2.5$-fold. Glycolytic enzymes - More than 10 enzymes related to glycolysis, the pentose phosphate pathway, glycogen metabolism and amino acid synthesis were all upregulated in MCF7-WNT1/FGF3 cells. Protein synthesis machinery - Over 35 proteins related to protein synthesis, including ribosome-related proteins, enzymes for tRNA synthesis, chaperones for protein folding and amino acid transporters, were all up upregulated in MCF7-WNT1/ FGF3 cells. EMT markers - Greater than 45 proteins known to be associated with the EMT phenotype were upregulated in MCF7-WNT1/FGF3 cells. Examples include FRS2 (FGF receptor substrate-2; $>10$-fold) and $\beta$-catenin ( $>2$-fold).
MCF7-WNT1/FGF3 cells show a functional increase in mitochondrial mass and mitochondrial membrane potential

To further validate the mitochondrial phenotype of MCF7-WNT1/FGF3 cells, we used fluorescent probes to quantitate mitochondrial mass and mitochondrial membrane potential by FACS analysis. For this purpose, we used MitoTracker Deep-Red (640-nm) to measure mitochondrial mass and MitoTracker Orange (561-nm), as a measure of mitochondrial membrane potential.

Figure 5 (Left panels) show that as compared to EV control MCF7 cells, MCF7 cells overexpressing WNT1/FGF3 show a clear shift to the right, for both mitochondrial mass and membrane potential. Furthermore, quantitation of fluorescence intensity (MFI) reveals that both of these mitochondrial parameters are significantly elevated in MCF7-WNT1/FGF3 cells (Figure 5 (Right panels)).

These results suggest that increased mitochondrial mass and function may be important features of the anabolic CSC phenotype.

\section{High mitochondrial mass is a key determinant of mammosphere-forming activity in parental MCF7 cells}

Based on our above observations with WNT1/ FGF3 signaling, we would predict that mitochondrial biogenesis is critical for mammosphere forming activity. To test this hypothesis more directly, we metabolically fractionated untransfected parental MCF7 cells, using MitoTracker Deep-Red, as a measure of mitochondrial mass. In this context, we chose to analyze three distinct metabolic phenotypic sub-groups: i) negative cells (little or no positive staining; mito-negative group); ii) bottom $5 \%$ (mito-low group); and top 5\% (mito-high group). Only live cells in each group were selected for this analysis.

Five thousand live cells from each group were then seeded per well, in 6-well low attachment plates, to measure mammosphere-forming efficiency. Remarkably, Figures 6 and 7 directly show that increasing mitochondrial mass results in a 3.0 to 5.5 -fold increase in mammosphereforming activity, depending on which gating parameters are used (singlet-gating vs. all live cells). A comparison with all live cells is also shown because mammary stem cells tend to be larger than non-stem cells (See Ref \# 37 for a discussion of this point).

As such, the mito-negative group showed the least 3-D sphere-forming activity, while the mito-high group showed the highest 3-D sphere-forming efficiency. Thus, we conclude that mitochondrial mass can be used to enrich for stem-like cancer cells that are able to undergo anchorage-independent propagation, under lowattachment conditions. 


\section{DISCUSSION}

The mouse mammary tumor virus (MMTV) initiates mammary tumorigenesis in mice by promoter insertion adjacent to two main integration sites, namely Int-1 (Wnt1) and Int-2 (Fgf3), driving the propagation of mammary cancer stem cells [11-13, 20]. Here, we developed an isogenic cell model of MMTV signaling to facilitate protein biomarker discovery in breast cancer (Figure 8). More specifically, we over-expressed WNT1 and FGF3 in MCF7 cells, an ER(+) human breast cancer cell line. Importantly, MCF7 cells overexpressing both WNT1 and FGF3 showed a 3.5 -fold increase in mammosphere formation. Proteomic analysis of this model system revealed the induction of EMT markers, mitochondrial proteins, glycolytic enzymes and protein synthesis machinery, consistent with an anabolic phenotype. The WNT1/FGF3-induced increases in mitochondrial function were validated by MitoTracker staining. Proteins up-regulated by WNT/FGF-signaling in MCF7 cells, were also transcriptionally over-expressed in breast cancer cells in vivo, based on the bioinformatic analysis of public datasets of laser-captured epithelial tumor cells from breast cancer patients. We believe that this isogenic cell model will accelerate the identification of new protein biomarkers to predict clinical outcomes in breast cancer patients. Remarkably, metabolic fractionation of parental MCF7 cells, via MitoTracker staining, indicated that mitochondrial mass is a key determinant of mammosphere-forming activity. The mitonegative group showed the least sphere-forming activity, while the mito-high group showed the highest sphereforming efficiency. Thus, new mitochondrial biogenesis is critical for the successful propagation of stem-like cancer cells.
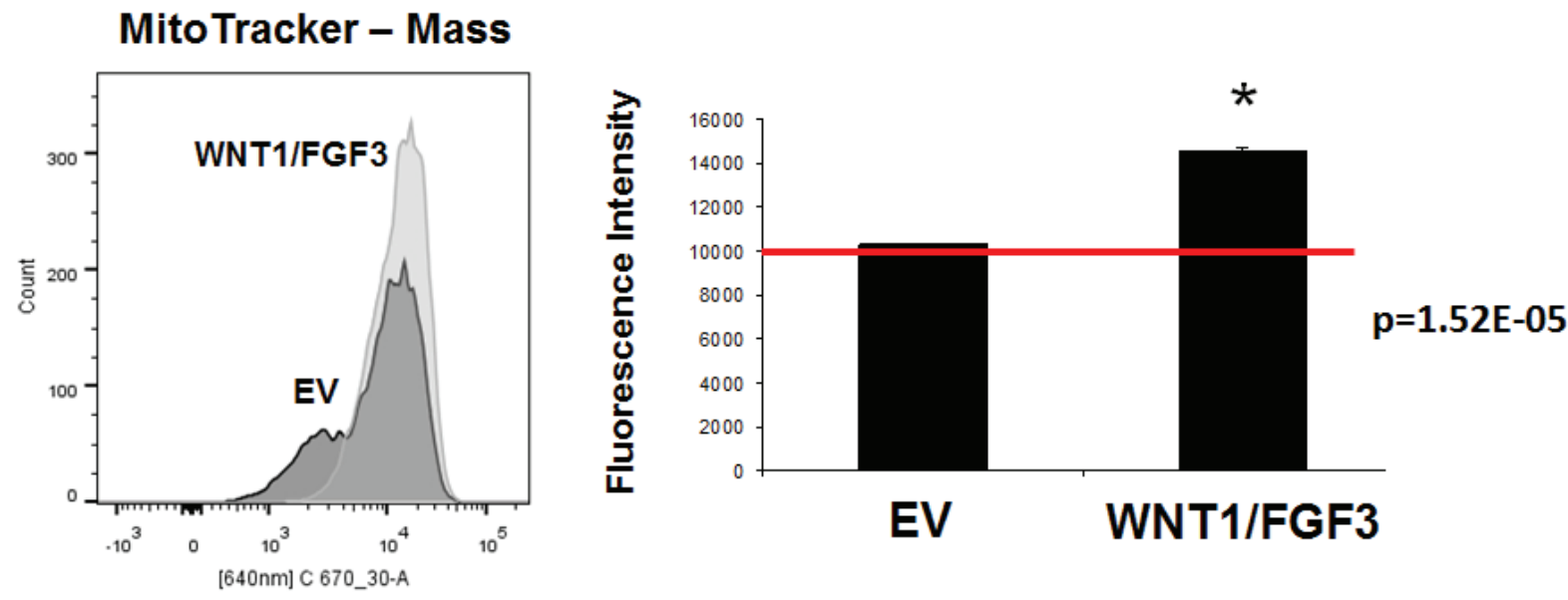

\section{MitoTracker - Activity}
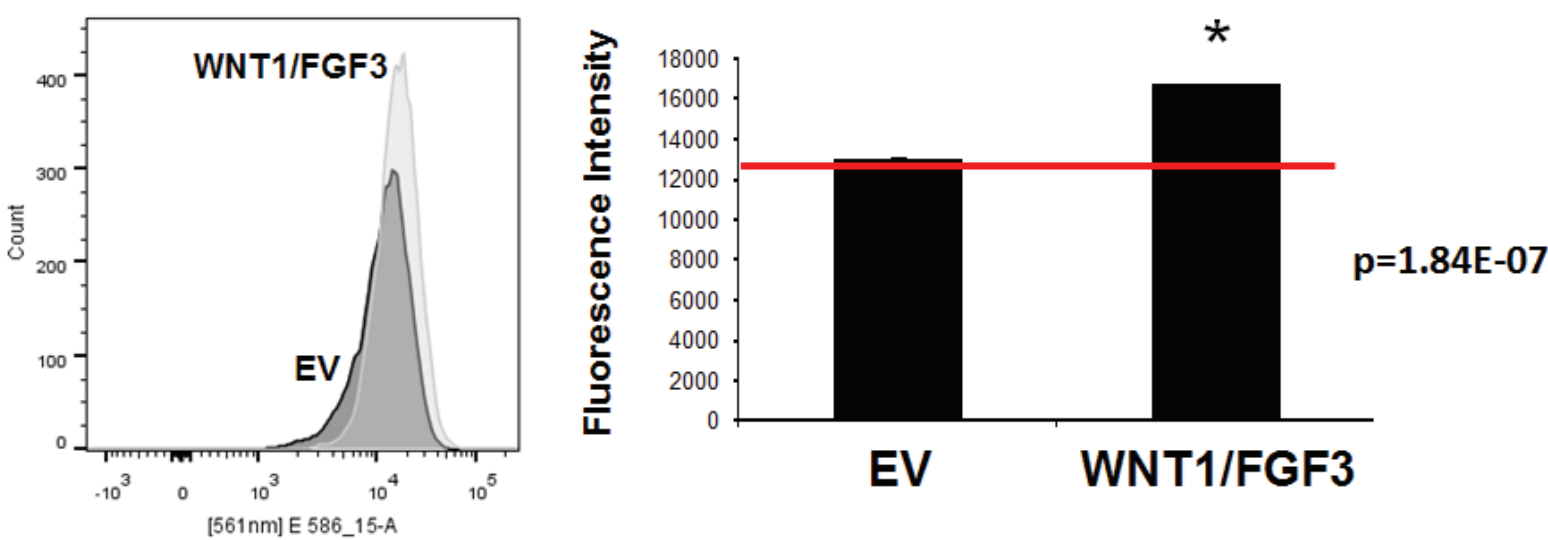

Figure 5: WNT1/FGF3 over-expressing MCF7 cells have increased mitochondrial mass and activity. We used two different fluorescent probes to quantitate mitochondrial mass and mitochondrial membrane potential by FACS analysis. Briefly, we employed MitoTracker Deep-Red (640-nm) to measure mitochondrial mass and MitoTracker Orange (561-nm), as a measure of mitochondrial membrane potential. Note that as compared to EV control MCF7 cells, MCF7 cells overexpressing WNT1/FGF3 show a clear shift to the right, for both mitochondrial mass (Lower panels) and membrane potential (Upper panels). Quantitation of fluorescence intensity (MFI) reveals that both of these mitochondrial parameters are significantly elevated in MCF7-WNT1/FGF3 cells. P-values are as shown. These results suggest that both mitochondrial mass and function may be critical features of the CSC phenotype. 
Table 5: WNT1/FGF3 Targets Increased in Human Breast Cancer Cells in Vivo: Mitochondria and Glycolysis.

\begin{tabular}{|c|c|c|c|}
\hline Symbol & Description & Fold-Change & P-value \\
\hline \multicolumn{4}{|c|}{ Mitochondrial-related Proteins/TCA Cycle (26) } \\
\hline$\overline{\text { ATP5O }}$ & ATP synthase subunit $O$, mitochondrial & 5.12 & 2.13E-06 \\
\hline ATP5B & ATP synthase subunit beta, mitochondrial & 5.04 & $2.75 \mathrm{E}-06$ \\
\hline ATP5A1 & ATP synthase subunit alpha, mitochondrial & 5.01 & 3.09E-06 \\
\hline COX6A1 & Cytochrome $\mathrm{c}$ oxidase subunit $6 \mathrm{~A}$, mitochondrial & 4.46 & 2.07E-05 \\
\hline ECHS1 & Enoyl-CoA hydratase, mitochondrial & 4.05 & $8.22 \mathrm{E}-05$ \\
\hline MDH1 & Malate dehydrogenase, cytoplasmic & 3.99 & $9.88 \mathrm{E}-05$ \\
\hline PCK2 & Phosphoenolpyruvate carboxykinase [GTP], mitochondrial & 3.88 & $1.43 \mathrm{E}-04$ \\
\hline SCD & Acyl-CoA desaturase & 3.70 & $2.55 \mathrm{E}-04$ \\
\hline HSPA9 & Stress-70 protein, mitochondrial & 3.69 & 2.64E-04 \\
\hline NQO1 & $\mathrm{NAD}(\mathrm{P}) \mathrm{H}$ dehydrogenase [quinone] 1 & 3.49 & $4.81 \mathrm{E}-04$ \\
\hline HSPD1 & $60 \mathrm{kDa}$ heat shock protein, mitochondrial & 3.42 & $5.93 E-04$ \\
\hline COX4l1 & Cytochrome $c$ oxidase subunit 4 isoform 1 , mitochondrial & 3.39 & $6.61 \mathrm{E}-04$ \\
\hline TUFM & Elongation factor Tu, mitochondrial & 3.38 & $6.74 \mathrm{E}-04$ \\
\hline C21orf33 & ES1 protein homolog, mitochondrial & 3.31 & $8.40 \mathrm{E}-04$ \\
\hline NDUFS1 & Mitochondrial NADH-ubiquinone oxidoreductase $75 \mathrm{kDa}$ subunit & 3.20 & $1.15 \mathrm{E}-03$ \\
\hline IDH1 & Isocitrate dehydrogenase [NADP] 1 & 3.18 & $1.22 \mathrm{E}-03$ \\
\hline OAT & Ornithine aminotransferase, mitochondrial & 3.17 & $1.25 \mathrm{E}-03$ \\
\hline CS & Citrate synthase, mitochondrial & 2.66 & $5.13 \mathrm{E}-03$ \\
\hline AK2 & Adenylate kinase 2, mitochondrial & 2.20 & $1.59 \mathrm{E}-02$ \\
\hline IDH3A & Isocitrate dehydrogenase [NAD] subunit alpha, mitochondrial & 2.16 & $1.78 \mathrm{E}-02$ \\
\hline PRKDC & DNA-dependent protein kinase catalytic subunit (maintains mt-DNA copy number) & 2.14 & $1.85 \mathrm{E}-02$ \\
\hline CLPX & ATP-dependent Clp protease ATP-binding subunit clpX-like, mitochondrial & 2.11 & $1.96 \mathrm{E}-02$ \\
\hline ABAT & 4-aminobutyrate aminotransferase, mitochondrial & 2.08 & $2.14 \mathrm{E}-02$ \\
\hline $\mathrm{ACO} 2$ & Aconitase 2, mitochondrial & 1.83 & 3.64E-02 \\
\hline DUT & Deoxyuridine 5 '-triphosphate nucleotidohydrolase, mitochondrial & 1.87 & 3.37E-02 \\
\hline ETFA & Electron transfer flavoprotein subunit alpha, mitochondrial & 1.76 & 4.25E-02 \\
\hline \multicolumn{4}{|c|}{ Enzymes Related to Glycolysis, the Pentose Phosphate Pathway, Glycogen, and Amino Acid Synthesis (Serine/Arginine) (4) } \\
\hline PKM2 & Pyruvate kinase & 3.26 & $9.79 \mathrm{E}-04$ \\
\hline PGK1 & Phosphoglycerate kinase & 2.46 & 8.66E-03 \\
\hline TKT & Transketolase & 2.20 & 1.60E-02 \\
\hline ENO1 & Enolase, alpha & 1.96 & $2.75 \mathrm{E}-02$ \\
\hline
\end{tabular}

-Transcriptional profiling data derived from the analysis of $\mathrm{N}=\mathbf{2 8}$ breast cancer patients are shown, high-lighting the levels of foldupregulation observed in the epithelial cancer cell compartment (relative to the tumor stroma), and corresponding p-values derived from the analysis of these clinical samples.

\section{FACS GATING: SINGLETS ONLY}

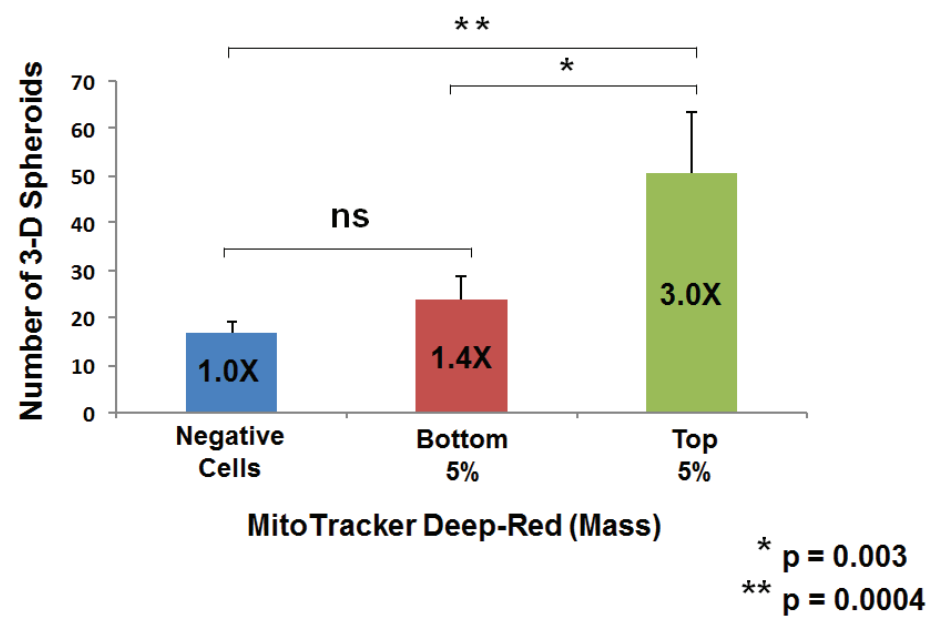

Figure 6: Metabolic fractionation of parental MCF7 cells directly correlates with mammosphere-forming activity: Gating for singlet cells. We metabolically fractionated parental MCF7 cells, using MitoTracker Deep-Red, as a measure of mitochondrial mass. In this context, we chose to analyze three distinct metabolic phenotypic groups: i) negative cells (little or no positive staining; mitonegative group); ii) bottom 5\% (mito-low group); and top 5\% (mito-high group). Only live cells in each group were selected for this analysis. Five thousand live cells from each group were then seeded per well, in 6-well low attachment plates, to measure mammosphereforming efficiency. Note that increasing mitochondrial mass results in a 3.0-fold increase in mammosphere-forming activity. Thus, the mito-deficient group showed the least sphere-forming activity, while the mito-high group showed the highest sphere-forming efficiency. Assays were performed in triplicate and repeated three times independently. The mean number of mammospheres (3-D spheroids) formed is shown. 
Table 6: WNT1/FGF3 Targets Increased in Human Breast Cancer Cells in Vivo: The EMT and Cell Migration.

\begin{tabular}{|c|c|c|c|}
\hline Symbol & Description & Fold-Change & P-value \\
\hline \multicolumn{4}{|c|}{ EMT Markers, Extracellular Matrix, Cell Migration and Cytoskeletal proteins (15) } \\
\hline$\overline{F L N B}$ & Filamin-B & 4.81 & $6.21 \mathrm{E}-06$ \\
\hline TPT1 & Translationally-controlled tumor protein & 3.43 & $5.81 \mathrm{E}-04$ \\
\hline CDC42 & Cell division control protein 42 homolog & 3.11 & $1.48 \mathrm{E}-03$ \\
\hline S100A11 & Protein S100-A11 & 2.88 & $2.81 \mathrm{E}-03$ \\
\hline ANXA2 & Annexin A2 & 2.83 & $3.28 \mathrm{E}-03$ \\
\hline MYOF & Myoferlin & 2.67 & $5.00 \mathrm{E}-03$ \\
\hline TUBB2A & Tubulin beta-2A chain & 2.63 & $5.56 \mathrm{E}-03$ \\
\hline SEPT2 & Septin-2 & 2.56 & $6.60 \mathrm{E}-03$ \\
\hline TAGLN2 & Transgelin-2 & 2.42 & 9.47E-03 \\
\hline IQGAP1 & IQ motif containing GTPase activating protein 1 (scaffold protein for CDC42) & 2.32 & 1.19E-02 \\
\hline HMGB1 & High mobility group protein B1 & 2.21 & 1.57E-02 \\
\hline CAPZB & F-actin-capping protein subunit beta & 2.19 & 1.66E-02 \\
\hline CDV3 & Carnitine deficiency-associated gene expressed in cardiac ventricle 3 & 2.04 & $2.30 \mathrm{E}-02$ \\
\hline FAM82B & Regulator of microtubule dynamics protein 1 & 1.97 & $2.72 \mathrm{E}-02$ \\
\hline MYH10 & Myosin, heavy polypeptide 10 , non-muscle & 1.82 & 3.69E-02 \\
\hline \multicolumn{4}{|c|}{ Miscellaneous (11) } \\
\hline PON2 & Paraoxonase 2, isoform & 4.02 & $9.25 \mathrm{E}-05$ \\
\hline MATR3 & Matrin-3 & 3.45 & $5.56 \mathrm{E}-04$ \\
\hline SH3BGRL & SH3 domain-binding glutamic acid-rich-like protein & 3.12 & $1.43 \mathrm{E}-03$ \\
\hline AHNAK & Neuroblast differentiation-associated protein, AHNAK & 2.57 & $6.41 \mathrm{E}-03$ \\
\hline CAST & Calpastatin A & 2.54 & $7.08 \mathrm{E}-03$ \\
\hline SEC24A & Protein transport protein Sec24A & 2.19 & $1.65 \mathrm{E}-02$ \\
\hline PABPC4 & Polyadenylate-binding protein 4 & 2.15 & $1.78 \mathrm{E}-02$ \\
\hline COMT & Soluble catechol-O-methyltransferase & 2.10 & 2.04E-02 \\
\hline STUB1 & E3 ubiquitin-protein ligase CHIP & 1.95 & $2.79 \mathrm{E}-02$ \\
\hline TFF1 & Trefoil factor 1 & 1.76 & 4.17E-02 \\
\hline HUWE1 & E3 ubiquitin-protein ligase HUWE1 & 1.75 & 4.33E-02 \\
\hline
\end{tabular}

-Transcriptional profiling data derived from the analysis of $\mathrm{N}=\mathbf{2 8}$ breast cancer patients are shown, high-lighting the levels of foldupregulation observed in the epithelial cancer cell compartment (relative to the tumor stroma), and corresponding $p$-values derived from the analysis of these clinical samples.

\section{FACS GATING: ALL LIVE CELLS}

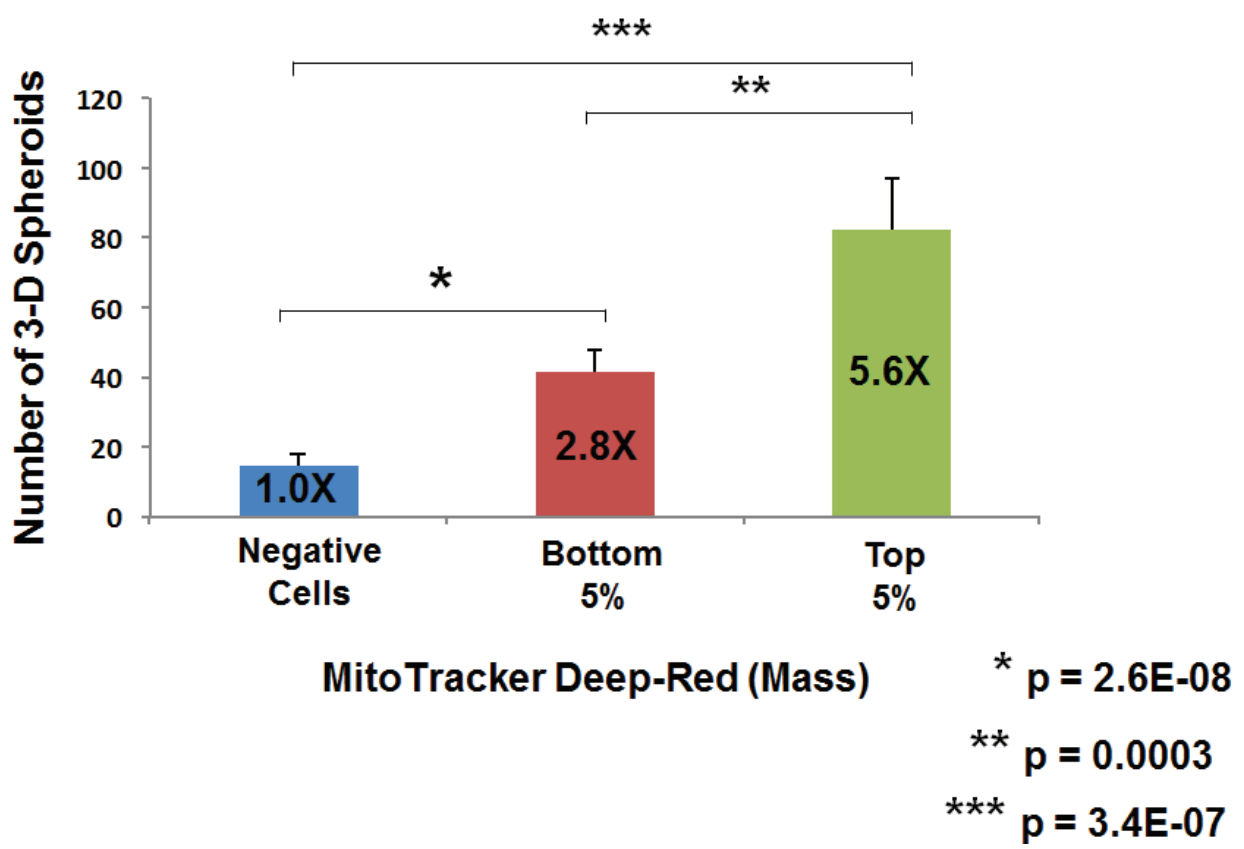

Figure 7: Metabolic fractionation of parental MCF7 cells directly correlates with mammosphere-forming activity: Gating for all live cells. As in Figure 6, except that FACS gating included all live cells, not only live singlets. Under these conditions, note that increasing mitochondrial mass results in a $>5.5$-fold increase in mammosphere-forming activity. Assays were performed in triplicate and repeated three times independently. The mean number of mammospheres (3-D spheroids) formed is shown. 
Table 7: WNT1/FGF3 Targets Increased in Human Breast Cancer Cells in Vivo: Ribosomes and Protein Synthesis.

\begin{tabular}{|c|c|c|c|}
\hline Symbol & Description & Fold-Change & P-value \\
\hline \multicolumn{4}{|c|}{ Ribosome-related proteins (8) } \\
\hline SRPRB & Signal recognition particle receptor subunit beta & 4.68 & 9.97E-06 \\
\hline RPL15 & 605 ribosomal protein L15 & 4.60 & $1.28 \mathrm{E}-05$ \\
\hline RPL13 & $60 S$ ribosomal protein L19 & 4.48 & 1.98E-05 \\
\hline RPL13 & $60 S$ ribosomal protein $\mathrm{L} 13$ & 4.48 & $1.98 \mathrm{E}-05$ \\
\hline RPL14 & 60 S ribosomal protein $\mathrm{L} 14$ & 4.45 & $2.15 \mathrm{E}-05$ \\
\hline RPS5 & 40S ribosomal protein S5 & 4.41 & $2.45 \mathrm{E}-05$ \\
\hline RPL4 & $60 S$ ribosomal protein $\mathrm{L} 4$ & 3.05 & 1.79E-03 \\
\hline NPM1 & NPM1 protein & 2.42 & $9.50 \mathrm{E}-03$ \\
\hline \multicolumn{4}{|c|}{ Translation initiation factors $(3)$} \\
\hline EIF2S1 & Eukaryotic translation initiation factor 2 , subunit 1 alpha, 35kDa & 3.98 & 1.04E-04 \\
\hline EIF3D & Eukaryotic translation initiation factor 3 subunit D & 2.85 & 3.13E-03 \\
\hline EIF5B & Eukaryotic translation initiation factor 5B & 2.58 & $6.29 \mathrm{E}-03$ \\
\hline \multicolumn{4}{|c|}{ Elongation factors (4) } \\
\hline EEF1B2 & Elongation factor 1-beta & 4.08 & 7.56E-05 \\
\hline EEF1G & Elongation factor 1-gamma & 3.71 & 2.44E-04 \\
\hline TUFM & Elongation factor Tu, mitochondrial & 3.38 & $6.74 \mathrm{E}-04$ \\
\hline EEF1D & Elongation factor 1-delta & 2.50 & 7.67E-03 \\
\hline \multicolumn{4}{|c|}{ Enzymes for tRNA synthesis (4) } \\
\hline C22orf28 & tRNA-splicing ligase RtcB homolog & 4.59 & 1.37E-05 \\
\hline EPRS & Bifunctional aminoacyl-tRNA synthetase (Glutamyl-Prolyl-tRNA Synthetase) & 4.06 & 8.10E-05 \\
\hline DARS & Aspartate--tRNA ligase, cytoplasmic & 3.43 & 5.87E-04 \\
\hline WARS & Tryptophan--tRNA ligase, cytoplasmic & 2.48 & 8.17E-03 \\
\hline \multicolumn{4}{|c|}{ Protein folding chaperones (heat shock proteins) (11) } \\
\hline HSP90AB1 & Heat shock protein HSP 90-beta & 4.94 & 4.03E-06 \\
\hline PPIA & Peptidyl-prolyl cis-trans isomerase A & 4.29 & 3.74E-05 \\
\hline CANX & Calnexin & 3.99 & $9.88 \mathrm{E}-05$ \\
\hline PDIA6 & Protein disulfide-isomerase $\mathrm{A} 6$ & 3.62 & 3.22E-04 \\
\hline HSPD1 & $60 \mathrm{kDa}$ heat shock protein, mitochondrial & 3.42 & 5.93E-04 \\
\hline PPIB & Peptidyl-prolyl cis-trans isomerase B & 3.28 & $9.25 \mathrm{E}-04$ \\
\hline HSPH1 & Heat shock protein $105 \mathrm{kDa}$ & 3.18 & $1.22 \mathrm{E}-03$ \\
\hline HSPA8 & Heat shock cognate $71 \mathrm{kDa}$ protein & 3.11 & 1.49E-03 \\
\hline PDIA3 & Protein disulfide-isomerase $\mathrm{A} 3$ & 2.53 & $7.22 \mathrm{E}-03$ \\
\hline HSP90B1 & Endoplasmin & 2.43 & $9.33 \mathrm{E}-03$ \\
\hline PDIA4 & Protein disulfide-isomerase A4 & 2.13 & $1.89 \mathrm{E}-02$ \\
\hline
\end{tabular}

-Transcriptional profiling data derived from the analysis of $\mathrm{N}=\mathbf{2 8}$ breast cancer patients are shown, high-lighting the levels of foldupregulation observed in the epithelial cancer cell compartment (relative to the tumor stroma), and corresponding p-values derived from the analysis of these clinical samples.

Figure 8: Anabolic CSC signaling: Exploiting a humanized model of MMTV signaling to identify the characteristics of anabolic CSCs and achieve the goals of personalized medicine. A humanized isogenic model of MMTV-signaling was generated by co-expressing WNT1 and FGF3 in MCF7 cells, an ER(+) human breast cancer cell line. This model was first validated using the mammosphere assay to measure stem cell activity and then subjected to unbiased labelfree proteomics analysis. WNT1/FGF3 protein targets identified in this manner were found to be transcriptionally over-expressed in human breast cancer cells in vivo, providing clinical validation of the success of our approach. Thus, we established that the anabolic CSC phenotype is characterized by the induction of EMT markers, mitochondrial proteins, glycolytic enzymes and protein synthesis machinery. These represent new classes of identified protein targets for drug discovery and the identification of companion diagnostics, to eradicate anabolic CSCs.

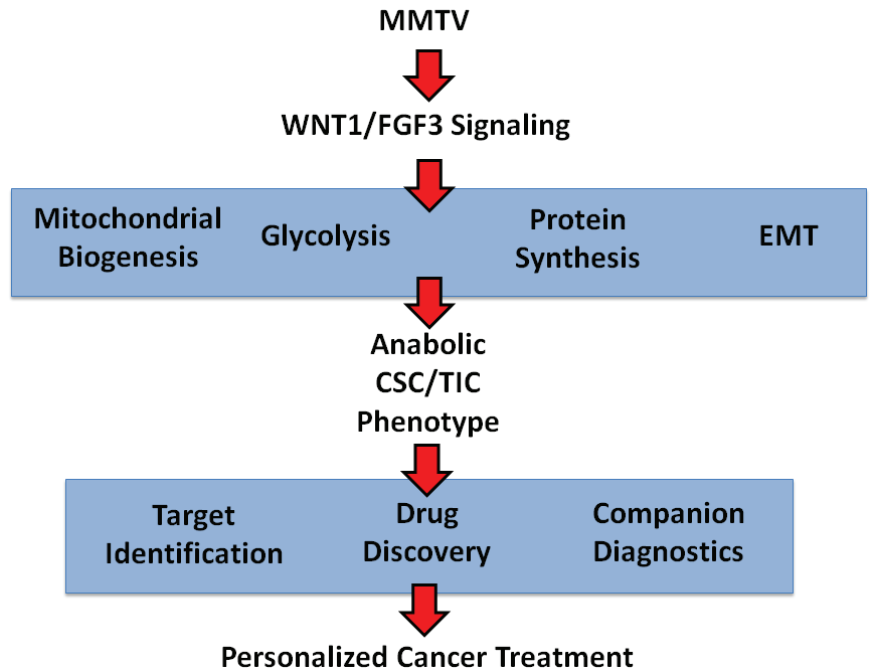


Role of new mitochondrial biogenesis in WNTsignaling and asymmetric cell division in stem cells

Interestingly, two previous studies have also linked WNT signaling to new mitochondrial biogenesis, in the context of skeletal muscle function and osteoblastic differentiation [24, 25]. For example, Yoon et al performed an si-RNA screen to identify novel protein targets that are critical for driving mitochondrial biogenesis in skeletal muscle cells [24]. For this purpose, they screened the effects of si-RNAs on $\mathrm{C} 2 \mathrm{C} 12$ cells, representing $>6,300$ genes, using a high-throughput FACS-based assay to measure mitochondrial function. Overall, they identified $>150$ proteins not previously recognized to be involved in the regulation of mitochondrial biogenesis. Bioinformatics analysis of this data set identified WNT/ $\beta$-catenin signaling as a key regulator of mitochondrial biogenesis. This was functionally validated by using si-RNAs targeting $\beta$-catenin and Axin2. Moreover, treatment of $\mathrm{C} 2 \mathrm{C} 12$ cells with Wnt3a increased mitochondrial biogenesis by nearly 2 -fold, which also directly correlated with a functional increase in oxygen consumption. Expression of a dominant-negative form of TCF4 blocked the effects of Wnt3a on mitochondrial biogenesis, indicating that the canonical Wnt-pathway was responsible for the metabolic effects of Wnt3a. Interestingly, Wnt3a mediated mitochondrial biogenesis also appeared to be dependent

Viral Promoter Insertion and Energy Metabolism

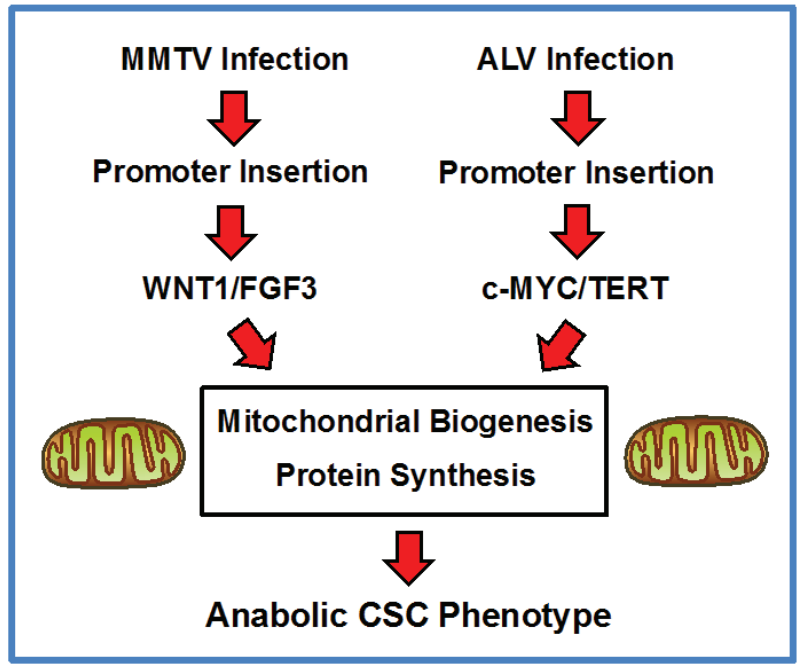

Figure 9: Convergent role of energy metabolism in the pathogenesis of viral oncogenesis, driven by promoter insertion: A new metabolic hypothesis. We propose that MMTV and ALV may induce oncogenesis by a convergent metabolic mechanism, which relies on an anabolic CSC phenotype, characterized by increased mitochondrial biogenesis and augmented protein synthesis. See the Discussion section for further details. ALV, avian leukosis virus; MMTV, mouse mammary tumor virus. on down-stream effectors, such as IRS-1 and c-MYC [24]. Therefore, the effects of WNT/ $\beta$-catenin signaling on mitochondrial biogenesis, may ultimately be mediated by the c-MYC proto-oncogene.

Asymmetric cell division is required for the maintenance of the stem cell phenotype and also occurs in stem-like cancer cells. Recently, Weinberg and Sabatini assessed how mitochondria are apportioned during asymmetric cell division, using an immortalized model of mammary epithelial stem cells [26]. Interestingly, they observed that "newly-synthesized" mitochondria were concentrated in stem cells during asymmetric cell division, while "old" mitochondria were segregated into daughter cells. As such, asymmetric cell division requires new mitochondrial biogenesis, for the propagation the stem cell phenotype. These findings could mechanistically explain our current results, that high-mitochondrial mass (Figures 6 and 7) directly correlates with "stemness" and mammosphere-forming efficiency.

\section{Role of mitochondrial biogenesis in anchorage- independent growth, in transformed fibroblasts and CSCs}

In 1984, Klebe and Harriss described the use of a colorimetric tetrazolium dye, namely MTT, to distinguish between normal and transformed fibroblasts, when the two different immortal isogenic cell lines were co-cultured [27]. More specifically, they showed that SV40-transformed BALB/3T3 fibroblasts, which were undergoing anchorage-independent growth (fociformation), were highly MTT-positive [27]. In contrast, non-transformed quiescent BALB/3T3 cells were MTTnegative, showing little or no staining [27]. As MTTstaining has largely been attributed to mitochondrial oxidative function and redox activity, these results may be the first description of an association between anchorageindependent growth and mitochondrial function.

In accordance with these findings, Fisher et al., 2011 [28] demonstrated that PGC1- $\alpha$ mediated activation of mitochondrial biogenesis is indeed required for the anchorage-independent growth of RAS-transformed fibroblasts. Moreover, recent studies with XCT790, a chemical inhibitor of the ERR- $\alpha /$ PGC1- $\alpha$ signaling network, directly showed that blocking mitochondrial biogenesis is sufficient to effectively prevent the anchorage-independent survival and propagation of epithelial CSCs [29]. Quantitatively similar results were also obtained with azithromycin and doxycycline [29, 31], two well-established antibiotic inhibitors of mitochondrial biogenesis, which target mitochondrial protein translation.

Thus, our current results are also consistent with the idea that mitochondrial power somehow helps to energize anchorage-independent growth, which is a key characteristic of CSCs. 


\section{Viral oncogenesis, promoter insertion and energy metabolism}

MMTV is known to cause the development of mammary tumors by promoter insertion proximal to cellular proto-oncogenes, that when over-expressed, confer an oncogenic phenotype. This appears to be largely through the constitutive activation of $\mathrm{WNT} / \beta$-catenin signaling. Here, we show that this signal transduction process also leads to the activation of mitochondrial biogenesis and an increase in the machinery necessary for protein synthesis, which is characteristic of an anabolic CSC phenotype. Previous studies have also shown that the ability of WNT/ $\beta$-catenin signaling to increase mitochondrial biogenesis is dependent on c-MYC activation, but in the context of skeletal muscle cells [24]. In further support of these ideas, MMTV Int-6 is eukaryotic translation initiation factor 3 (eIF3), which serves as a scaffolding protein to increase protein synthesis (Table 1).

Avian leukosis virus (ALV) is another pathogen that induces cancer, via a promoter insertion-based mechanism $[32,33]$. More specifically, ALV infection leads to proviral intergration and promoter insertion, driving the development of myeloid leukosis and, ultimately, frank leukemia in chickens. Interestingly, the most common ALV integration sites include c-MYC and hTERT, as well as other gene products related to mitochondrial biogenesis and function (NDUFS6 and PARK2) [34].

Taken together, these data imply that MMTV and ALV may induce oncogenesis by a convergent metabolic mechanism, which relies on the down-stream activation of c-MYC, driving increased mitochondrial biogenesis. Interestingly, c-MYC is also known to increase protein synthesis, by targeting translation initiation, as well as by directly increasing ribosomal biogenesis $[35,36]$. As such, MMTV and ALV may both ultimately induce the anabolic CSC phenotype, via increased mitochondrial biogenesis and increased protein synthesis (Figure 9). In further support of this idea, hTERT over-expression also appears to be directly associated with an anabolic CSC phenotype, driving increased mitochondrial biogenesis and augmented protein synthesis [37]. Thus, this intriguing hypothesis, regarding the existence of a convergent metabolic mechanism, underlying MMTV and ALV oncogenesis, undoubtedly deserves further study.

\section{Mitochondrial DNA content and mitochondrial mass both increase during the transition from normal tissue to hyperplasia and malignancy}

Interestingly, previous studies in human endometrial cancer have monitored i) mt-DNA content (by RT-PCR) and ii) mitochondrial mass (using the enzyme activity of citrate synthase), during the transition to malignancy. More specifically, they observed that both of these parameters increased by up to 2 to 3 fold, when normal endometrial tissue was directly compared to endometrial cancer [38]. Similarly, they also observed that the protein expression levels of three mitochondrial-related transcription factors (TFAM, NRF1 and PGC1-alpha) were all significantly increased by nearly 2 -fold [39]. Taken together, these results are all consistent with an increase in mitochondrial biogenesis, during the pathogenesis of tumor initiation.

Similarly, we have previously shown that markers of mitochondrial mass and mitochondrial activity are specifically localized to the basal stem cell layer in normal human mucosa, which co-localizes with Ki67, an established marker of cell proliferation [40]. In addition, this mitochondria-rich population of cells is dramatically expanded in head and neck cancers [40] and breast cancers [41-43]. Moreover, recombinant over-expression of mitochondrial-related proteins, such as PGC1-alpha/ beta, POLRMT, MitoNEET or GOLPH3, is sufficient to promote tumor growth, by up to 3 -fold, in xenografted pre-clinical models of human breast cancers [44, 45]. Finally, mitochondrial biogenesis and mass are also significantly increased in hematological malignancies, such as in chronic lymphocytic leukemia (CLL) and acute myeloid leukemia (AML) [46-49].

This increase in mitochondrial mass also appears to be part of a normal developmental process, as mitochondrial biogenesis increases between 25 to 50 fold, during mammalian embryogenesis, especially from the two-cell stage to the early blastocyst [50]. This early stage of embryogenesis reflects the proliferative expansion normal progenitor cells. Interestingly, pluripotent ES cell lines are derived from the inner cell mass of the blastocyst.

\section{CONCLUSIONS}

In summary, the use of mitochondrial mass as a surrogate metabolic biomarker of mitochondrial biogenesis allows for the identification of stem-like cancer cells, facilitating CSC enrichment for future biomarker studies and aiding in the design of novel therapeutic interventions. In this context, MCF7 cells over-expressing WNT1/FGF3 will provide a novel model system for these ongoing investigations.

\section{MATERIALS AND METHODS}

\section{Materials}

Breast cancer cell lines (MCF7 and T47D) were originally purchased from the ATCC. Gibco-brand cell culture media (DMEM and DMEM/F12) was purchased from Life Technologies. Lentiviral vectors encoding WNT1 [EX-B0110-Lv105(puro)] and FGF3 [EX- 
A0154-Lv151(neo)] were obtained commercially from Genecopoeia (USA), along with appropriate empty vector controls [EX-Neg-Lv105(puro) and EX-Neg-Lv151(neo)]. Antibodies directed against FGF3 (\# HPA012692, Sigma) and WNT1 (\# ab15251, Abcam) were also obtained commercially. MitoTracker probes (Deep-Red and Orange) were purchased from Molecular Probes, via Life Technologies.

\section{MCF7 cell viral transduction and antibiotic selection}

Lentiviral particles harboring human WNT1 [EXB0110-Lv105(puro)] or human FGF3 [EX-A0154Lv151(neo)] were prepared and used to stably transduce MCF7 cells, according to the manufacturer's protocol. After 24 hours, media containing the virus was removed and replaced with standard media. Cells were then selected with puromycin $(2 \mu \mathrm{g} / \mathrm{ml})$ or G418 $(2 \mathrm{mg} / \mathrm{ml})$, for up to 10 days. MCF7 cells harboring the empty vector alone controls were generated at the same time in parallel. MCF7-WNT1/FGF3 cells were generated by serial transduction with both WNT1 and FGF3 lentiviral vectors.

\section{WNT1 and FGF3 immunoblotting}

Transduced MCF7 cells were seeded in $10 \mathrm{~cm}$ dishes for $72 \mathrm{hrs}$. Then, cells were lysed in RIPA buffer (Sigma), containing proteinase inhibitors (Roche) and kept at $4{ }^{\circ} \mathrm{C}$ for 30 minutes. Lysates were collected by centrifugation for 10 minutes at $10,000 \mathrm{x} \mathrm{g}$, and protein concentration were determined using the BCA protein assay kit (Pierce). Samples were diluted into SDS-PAGE sample buffer and were boiled for 5 minutes before being separated by SDS-PAGE, using a $4-15 \%$ gradient Mini-PROTEAN TGX Gel (Biorad). Samples were then transferred onto a nitrocellulose membrane (Biorad), blocked in 5\% milk in TBS-Tween 20 (Sigma) and probed with antibodies directed against WNT1 or FGF3 and $\beta$-actin (Santa Cruz Biotechnology, \#sc-1616), using a secondary antibody at a dilution of 1 to 5000 . Bound antibodies were detected using the Supersignal West Pico Chemiluminiscent substrate (ThermoScientific). Alternatively, in the laboratory, blots were also routinely processed with a blocking solution containing BSA, as a blocking agent. Similarly, other comparable antibodies directed against $\beta$-actin were used, but were obtained from different commercial sources, such as Sigma.

\section{Assessment of mammosphere forming activity}

Mammosphere formation was carried out, essentially as described previously by Clarke and colleagues, without any significant modifications [51]. MCF7 cells were plated at a density of 500 cells $/ \mathrm{cm}^{2}$ in mammosphere medium in culture dishes coated with poly-HEMA (Sigma, \#P3932). After 5 days, 3D spheroids with a diameter greater than $50 \mu \mathrm{m}$ were counted using a microscope, fitted with a graticule eye-piece, and the percentage of cells which formed spheroids was calculated and normalized to one ( $1=100 \%$ MSE; mammosphere forming efficiency). Mammosphere assays were performed in triplicate and repeated three times independently.

\section{Conditioned media experiments}

One million MCF7 cells transfected with WNT1/ FGF3 or empty vector alone controls were plated for 24 hours in DMEM (10\% FCS). Cells were then washed in PBS, the subsequently cultured for 72 hours in $10 \mathrm{ml}$ of DMEM/F12 phenol-red free media (mammosphere media). Media was then collected and cells were removed by centrifugation at $1800 \mathrm{rpm}$ for 10 minutes. Conditioned media was then added directly to mammosphere assays of parental untransfected breast cancer cell lines (MCF7 and T47D) in a ratio of 1:1 with fresh mammosphere formation media.

\section{Unbiased label-free proteomics analysis}

Proteomics analysis was carried out essentially as we previously described, with minor modifications [52, 53]. Statistical analyses were performed using ANOVA and only fold-changes in proteins with a p-value less than 0.05 were considered significant. Unbiased proteomics and the statistical analysis of the results were performed by the Biological Mass Spectrometry Core Facility, at the Cancer Research UK Manchester Institute, under the supervision of Dr. Duncan L. Smith.

\section{Bioinformatics analysis with publically available human breast cancer clinical data}

To determine the possible translational significance of our proteomics analysis, we intersected our MCFbased WNT/FGF proteomics data with human genomewide transcriptional profiling data. These human clinical data were derived from publically available human breast cancer samples, in which breast cancer cells were separated by laser-capture microdissection from tumor stromal cells. Transcriptional profiles were analyzed from $\mathrm{N}=28$ human breast cancer patients [54].

\section{Analysis of mitochondrial mass and membrane potential}

To measure mitochondrial activity, cells were stained with MitoTracker Orange (\#M7510, Invitrogen), 
whose accumulation in mitochondria is dependent upon membrane potential. To measure mitochondrial mass, cells were stained with MitoTracker Deep Red (\#M22426, Invitrogen), localizing to mitochondria regardless of mitochondrial membrane potential. Cells were incubated with pre-warmed MitoTracker staining solution (diluted in PBS/CM to a final concentration of $10 \mathrm{nM}$ ) for $30-60$ min at $37{ }^{\circ} \mathrm{C}$. All subsequent steps were performed in the dark. Cells were washed in PBS, harvested, and resuspended in $300 \mu \mathrm{L}$ of PBS. Cells were then analyzed by flow cytometry. Data analysis was performed using FlowJo software.

\section{Metabolic fractionation of parental MCF7 cells using MitoTracker}

Using MitoTracker Deep-Red staining as a marker of mitochondrial mass, we metabolically fractionated parental MCF7 cells, using FACS analysis and cell collection. In these experiments, we analyzed three different metabolic sub-groups: i) negative cells (little or no positive staining; mito-negative group); ii) bottom $5 \%$ (mito-low group); and top 5\% (mito-high group). Only live cells in each group were selected for this analysis. Five thousand live cells from each group (performed in triplicate) were then seeded per well, in 6-well low attachment plates, to measure mammosphere-forming efficiency. Gating for cell size was varied to take into account the observation that stem-like mammary cells may be physically larger than "bulk" cancer cells, as was previously suggested.

This method is a further refinement of a protocol used by Farnie, Sotgia and Lisanti, in a companion study published in parallel [55]. Importantly, very similar results were obtained here, indicating that the method is operator independent. For example, see Figure 4 (Panel A) in Farnie et al., 2015, for comparison purposes [55]; however, in this companion paper, the mito-negative group was not analyzed.

\section{Statistical analyses}

Statistical significance was determined using the Student's t-test or ANOVA, where appropriate. Values of less than 0.05 were considered significant. Data in figures are shown as the mean \pm SEM, unless stated otherwise.

\section{ACKNOWLEDGEMENTS}

We thank the University of Manchester for providing start-up funds that contributed to the success of this study. The Sotgia and Lisanti Laboratories were supported, in part, by funding from the European Union (ERC Advanced Grant), Breast Cancer Now, and the Manchester Cancer Research Centre (MCRC). DLS was core-funded by CRUK. Dr. Ubaldo E. MartinezOutschoorn was supported by the National Cancer Institute (NCI) of the National Institutes of Health (NIH), under Award Number K08-CA175193-01A1. Drs. Bevilacqua, Mazzanti and McDonnell were supported by the Pisa Science Foundation.

\section{CONFLICTS OF INTEREST}

There is no conflict of interest.

\section{Author Contributions}

MPL initiated and coordinated this internationally collaborative project (UK, US and Italy). RL, MC, LW, DLS and GB performed all the experiments, analyzed the data and generated the figures with experimental data. MPL and FS wrote the first draft of the manuscript, which was extensively edited by the other authors, who also contributed ideas and suggestions for the discussion topics. RL also contributed heavily to the writing of the Material and Methods section and the figure legends. MPL generated the schematic summary diagrams. Unbiased proteomics and the statistical analysis of the proteomic results were performed by the Biological Mass Spectrometry Core Facility, at the Cancer Research UK Manchester Institute, under the supervision of DLS.

\section{Abbreviations}

CSCs, cancer stem-like cells; MMTV, mouse mammary tumor virus; TICs, tumor-initiating cells; EMT, epithelial-mesenchymal transition; ALV, avian leukosis virus; MTT, 3-(4,5-dimethylthiazol-2-yl)-2,5diphenyltetrazolium bromide

\section{REFERENCES}

1. Kilham L. Isolation in suckling mice of a virus from $\mathrm{C} 3 \mathrm{H}$ mice harboring Bittner milk agent. Science. 1952; 116:391392.

2. Moore R, Dixon M, Smith R, Peters G and Dickson C. Complete nucleotide sequence of a milk-transmitted mouse mammary tumor virus: two frameshift suppression events are required for translation of gag and pol. Journal of virology. 1987; 61:480-490.

3. Cohen JC. Methylation of milk-borne and genetically transmitted mouse mammary tumor virus proviral DNA. Cell. 1980; 19:653-662.

4. Cohen JC and Varmus HE. Proviruses of mouse mammary tumor virus in normal and neoplastic tissues from GR and C3Hf mouse strains. Journal of virology. 1980; 35:298-305.

5. Mazzanti CM, Lessi F, Armogida I, Zavaglia K, Franceschi S, Hamad MA, Roncella M, Ghilli M, Boldrini A, 
Aretini P, Fanelli G, Marchetti I, Scatena C, Hochman J, Naccarato AG and Bevilacqua G. Human saliva as route of inter-human infection for mouse mammary tumor virus. Oncotarget. 2015; 6: 18355-18363.

6. Mazzanti CM, Al Hamad M, Fanelli G, Scatena C, Zammarchi F, Zavaglia K, Lessi F, Pistello M, Naccarato AG and Bevilacqua G. A mouse mammary tumor virus envlike exogenous sequence is strictly related to progression of human sporadic breast carcinoma. The American journal of pathology. 2011; 179:2083-2090.

7. Nusse $R$, Theunissen $H$, Wagenaar E, Rijsewijk F, Gennissen A, Otte A, Schuuring E and van Ooyen A. The Wnt-1 (int-1) oncogene promoter and its mechanism of activation by insertion of proviral DNA of the mouse mammary tumor virus. Molecular and cellular biology. 1990; 10:4170-4179.

8. Nusse R, van Ooyen A, Cox D, Fung YK and Varmus $\mathrm{H}$. Mode of proviral activation of a putative mammary oncogene (int-1) on mouse chromosome 15. Nature. 1984; 307:131-136.

9. van Ooyen A and Nusse R. Structure and nucleotide sequence of the putative mammary oncogene int-1; proviral insertions leave the protein-encoding domain intact. Cell. 1984; 39:233-240.

10. Dickson C, Smith R, Brookes S and Peters G. Tumorigenesis by mouse mammary tumor virus: proviral activation of a cellular gene in the common integration region int-2. Cell. 1984; 37:529-536.

11. Li Y, Hively WP and Varmus HE. Use of MMTV-Wnt-1 transgenic mice for studying the genetic basis of breast cancer. Oncogene. 2000; 19:1002-1009.

12. Peters G, Brookes S, Smith R, Placzek M and Dickson C. The mouse homolog of the hst/k-FGF gene is adjacent to int- 2 and is activated by proviral insertion in some virally induced mammary tumors. Proceedings of the National Academy of Sciences of the United States of America. $1989 ; 86: 5678-5682$

13. Kwan H, Pecenka V, Tsukamoto A, Parslow TG, Guzman R, Lin TP, Muller WJ, Lee FS, Leder P and Varmus HE. Transgenes expressing the Wnt-1 and int-2 proto-oncogenes cooperate during mammary carcinogenesis in doubly transgenic mice. Molecular and cellular biology. 1992; 12:147-154.

14. Klaus A and Birchmeier W. Wnt signalling and its impact on development and cancer. Nature reviews Cancer. 2008; 8:387-398.

15. Cadigan KM and Nusse R. Wnt signaling: a common theme in animal development. Genes \& development. 1997; 11:3286-3305.

16. Hatch EP, Noyes CA, Wang X, Wright TJ and Mansour SL. Fgf3 is required for dorsal patterning and morphogenesis of the inner ear epithelium. Development. 2007; 134:36153625.

17. Katoh $\mathrm{M}$ and Katoh M. Cross-talk of WNT and FGF signaling pathways at GSK3beta to regulate beta-catenin and SNAIL signaling cascades. Cancer biology \& therapy. 2006; 5:1059-1064.

18. Katoh Y and Katoh M. FGF signaling inhibitor, SPRY4, is evolutionarily conserved target of WNT signaling pathway in progenitor cells. International journal of molecular medicine. 2006; 17:529-532.

19. Ross SR. Mouse mammary tumor virus molecular biology and oncogenesis. Viruses. 2010; 2:2000-2012.

20. Callahan R and Smith GH. Common integration sites for MMTV in viral induced mouse mammary tumors. Journal of mammary gland biology and neoplasia. 2008; 13:309321.

21. Callahan R and Smith GH. MMTV-induced mammary tumorigenesis: gene discovery, progression to malignancy and cellular pathways. Oncogene. 2000; 19:992-1001.

22. Cho RW, Wang X, Diehn M, Shedden K, Chen GY, Sherlock G, Gurney A, Lewicki J and Clarke MF. Isolation and molecular characterization of cancer stem cells in MMTV-Wnt-1 murine breast tumors. Stem cells. 2008; 26:364-371.

23. Mani SA, Guo W, Liao MJ, Eaton EN, Ayyanan A, Zhou AY, Brooks M, Reinhard F, Zhang CC, Shipitsin M, Campbell LL, Polyak K, Brisken C, Yang J and Weinberg RA. The epithelial-mesenchymal transition generates cells with properties of stem cells. Cell. 2008; 133:704-715.

24. Yoon JC, Ng A, Kim BH, Bianco A, Xavier RJ and Elledge SJ. Wnt signaling regulates mitochondrial physiology and insulin sensitivity. Genes \& development. 2010; 24:15071518.

25. An JH, Yang JY, Ahn BY, Cho SW, Jung JY, Cho HY, Cho YM, Kim SW, Park KS, Kim SY, Lee HK and Shin $\mathrm{CS}$. Enhanced mitochondrial biogenesis contributes to Wnt induced osteoblastic differentiation of C3H10T1/2 cells. Bone. 2010; 47:140-150.

26. Katajisto P, Dohla J, Chaffer CL, Pentinmikko N, Marjanovic N, Iqbal S, Zoncu R, Chen W, Weinberg RA and Sabatini DM. Stem cells. Asymmetric apportioning of aged mitochondria between daughter cells is required for stemness. Science. 2015; 348:340-343.

27. Klebe RJ and Harriss JV. A technically simple "nonlethal" vital staining procedure for viral plaque and cell transformation assays. Arch Virol. 1984; 81:359-62.

28. Fisher KW, Das B, Kortum RL, Chaika OV and Lewis RE. Kinase suppressor of ras 1 (KSR1) regulates PGC1 $\alpha$ and estrogen-related receptor $\alpha$ to promote oncogenic Rasdependent anchorage-independent growth. Mol Cell Biol. 2011; 31:2453-61.

29. De Luca A, Fiorillo M, Peiris-Pagès M, Ozsvari B, Smith DL, Sanchez-Alvarez R, Martinez-Outschoorn UE, Cappello AR, Pezzi V, Lisanti MP and Sotgia F. Mitochondrial biogenesis is required for the anchorageindependent survival and propagation of stem-like cancer cells. Oncotarget. 2015; 6:14777-95. 
30. Lamb R, Fiorillo M, Chadwick A, Ozsvari B, Reeves KJ, Smith DL, Clarke RB, Howell SJ, Cappello AR, MartinezOutschoorn UE, Peiris-Pagès M, Sotgia F and Lisanti MP. Doxycycline down-regulates DNA-PK and radiosensitizes tumor initiating cells: Implications for more effective radiation therapy. Oncotarget. 2015; 6:14005-25.

31. Lamb R, Ozsvari B, Lisanti CL, Tanowitz HB, Howell A, Martinez-Outschoorn UE, Sotgia F and Lisanti MP. Antibiotics that target mitochondria effectively eradicate cancer stem cells, across multiple tumor types: treating cancer like an infectious disease. Oncotarget. 2015; 6:456984.

32. Neel BG, Hayward WS, Robinson HL, Fang J and Astrin SM. Avian leukosis virus-induced tumors have common proviral integration sites and synthesize discrete new RNAs: oncogenesis by promoter insertion. Cell. 1981; 23:323-34.

33. Hayward WS, Neel BG and Astrin SM. Activation of a cellular onc gene by promoter insertion in ALV-induced lymphoid leukosis. Nature. 1981; 290: 475-80.

34. Li Y1, Liu X, Yang Z, Xu C, Liu D, Qin J, Dai M, Hao J, Feng M, Huang X, Tan L, Cao W and Liao M. The MYC, TERT, and ZIC1 genes are common targets of viral integration and transcriptional deregulation in avian leukosis virus subgroup J-induced myeloid leukosis. J Virol. 2014; 88:3182-91.

35. Meyer N and Penn LZ. Reflecting on 25 years with MYC. Nat Rev Cancer. 2008; 8: 976-90.

36. Schmidt EV. The role of c-myc in regulation of translation initiation. Oncogene. 2004; 23:3217-21.

37. Lamb R, Ozsvari B, Bonuccelli G, Smith DL, Pestell RG, Martinez-Outschoorn UE, Clarke RB, Sotgia F, and Lisanti MP. Dissecting tumor metabolic heterogeneity: Telomerase and large cell size metabolically define a sub-population of stem-like, mitochondrial-rich, cancer cells. Oncotarget, 2015; 6:21892-905. doi: 10.18632/oncotarget.5260.

38. Cormio A, Guerra F, Cormio G, Pesce V, Fracasso F, Loizzi V, Resta L, Putignano G, Cantatore P, Selvaggi LE, Gadaleta MN. Mitochondrial DNA content and mass increase in progression from normal to hyperplastic to cancer endometrium. BMC Res Notes. 2012; 5:279.

39. Cormio A, Guerra F, Cormio G, Pesce V, Fracasso F, Loizzi V, Cantatore P, Selvaggi L, Gadaleta MN. The PGC1alpha-dependent pathway of mitochondrial biogenesis is upregulated in type I endometrial cancer. Biochem Biophys Res Commun. 2009; 390:1182-5.

40. Curry JM, Tuluc M, Whitaker-Menezes D, Ames JA, Anantharaman A, Butera A, Leiby B, Cognetti DM, Sotgia F, Lisanti MP and Martinez-Outschoorn UE. Cancer metabolism, stemness and tumor recurrence: MCT1 and MCT4 are functional biomarkers of metabolic symbiosis in head and neck cancer. Cell Cycle; 12: 1371-84.

41. Whitaker-Menezes D1, Martinez-Outschoorn UE, Flomenberg N, Birbe RC, Witkiewicz AK, Howell A, Pavlides S, Tsirigos A, Ertel A, Pestell RG, Broda P, Minetti
C, Lisanti MP and Sotgia F. Hyperactivation of oxidative mitochondrial metabolism in epithelial cancer cells in situ: Visualizing the therapeutic effects of metformin in tumor tissue. Cell Cycle. 2011; 10: 4047-64.

42. Sotgia F, Whitaker-Menezes D, Martinez-Outschoorn UE, Flomenberg N, Birbe RC, Witkiewicz AK, Howell A, Philp NJ, Pestell RG and Lisanti MP. Mitochondrial metabolism in cancer metastasis: Visualizing tumor cell mitochondria and the "reverse Warburg effect" in positive lymph node tissue. Cell Cycle. 2012; 11:1445-54.

43. Ertel A, Tsirigos A, Whitaker-Menezes D, Birbe RC, Pavlides S, Martinez-Outschoorn UE, Pestell RG, Howell A, Sotgia F and Lisanti MP. Is cancer a metabolic rebellion against host aging? In the quest for immortality, tumor cells try to save themselves by boosting mitochondrial metabolism. Cell Cycle. 2012; 11:253-63.

44. Salem AF, Whitaker-Menezes D, Howell A, Sotgia F and Lisanti MP. Mitochondrial biogenesis in epithelial cancer cells promotes breast cancer tumor growth and confers autophagy resistance. Cell Cycle. 2012; 11:4174-80.

45. Salem AF, Whitaker-Menezes D, Lin Z, MartinezOutschoorn UE, Tanowitz HB, Al-Zoubi MS, Howell A, Pestell RG, Sotgia F and Lisanti MP. Twocompartment tumor metabolism: autophagy in the tumor microenvironment and oxidative mitochondrial metabolism (OXPHOS) in cancer cells. Cell Cycle. 2012;11:2545-56.

46. Jitschin R, Hofmann AD, Bruns H, Giessl A, Bricks J, Berger J, Saul D, Eckart MJ, Mackensen A, Mougiakakos D. Mitochondrial metabolism contributes to oxidative stress and reveals therapeutic targets in chronic lymphocytic leukemia. Blood. 2014; 123: 2663-72.

47. Jia L, Gribben JG. Dangerous power: mitochondria in CLL cells. Blood. 2014 ;123:2596-7.

48. Carew JS, Nawrocki ST, Xu RH, Dunner K, McConkey DJ, Wierda WG, Keating MJ, Huang P. Increased mitochondrial biogenesis in primary leukemia cells: the role of endogenous nitric oxide and impact on sensitivity to fludarabine. Leukemia. 2004; 18:1934-40.

49. Schimmer AD, Skrtić M. Therapeutic potential of mitochondrial translation inhibition for treatment of acute myeloid leukemia. Expert Rev Hematol. 2012; 5: 117-9.

50. Pikó L and Taylor KD. Amounts of mitochondrial DNA and abundance of some mitochondrial gene transcripts in early mouse embryos. Dev Biol. 1987;123:364-74.

51. Shaw FL, Harrison H, Spence K, Ablett MP, Simoes BM, Farnie $\mathrm{G}$ and Clarke RB. A detailed mammosphere assay protocol for the quantification of breast stem cell activity. Journal of mammary gland biology and neoplasia. 2012; 17:111-117.

52. Holland M, Castro FV, Alexander S, Smith D, Liu J, Walker M, Bitton D, Mulryan K, Ashton G, Blaylock M, Bagley S, Connolly Y, Bridgeman J, Miller C, Krishnan S, Dempsey $\mathrm{C}$, et al. RAC2, AEP, and ICAM1 expression are associated with $\mathrm{CNS}$ disease in a mouse model of pre-B childhood 
acute lymphoblastic leukemia. Blood. 2011; 118:638-649.

53. Lamb R, Harrison H, Hulit J, Smith DL, Lisanti MP, Sotgia F. Mitochondria as new therapeutic targets for eradicating cancer stem cells: Quantitative proteomics and functional validation via MCT1/2 inhibition. Oncotarget. 2014; 5:11029-37.

54. Casey T, Bond J, Tighe S, Hunter T, Lintault L, Patel O, Eneman J, Crocker A, White J, Tessitore J, Stanley M, Harlow S, Weaver D, Muss H and Plaut K. Molecular signatures suggest a major role for stromal cells in development of invasive breast cancer. Breast cancer research and treatment. 2009; 114:47-62.

55. Farnie G, Sotgia F and Lisanti MP. High mitochondrial mass identifies a sub-population of stem-like cancer cells that are chemo-resistant. Oncotarget 2015 Sep 30. doi: 10.18632/oncotarget.5401. 